\title{
$A b$ initio study of chemical disorder as an effective stabilizing mechanism of bcc-based TiAl(+Mo)
}

\author{
Neda Abdoshahi, ${ }^{1, *}$ Petra Spoerk-Erdely $\odot,{ }^{1}$ Martin Friák, ${ }^{2,3}$ Svea Mayer $\odot,{ }^{1}$ Mojmír Šob $\odot,{ }^{4,2,5}$ and David Holec $\circledast^{1, \dagger}$ \\ ${ }^{1}$ Department of Materials Science, Montanuniversität Leoben, Franz-Josef-Straße 18, A-8700 Leoben, Austria \\ ${ }^{2}$ Institute of Physics of Materials, Czech Academy of Sciences, Žižkova 22, CZ-616 62 Brno, Czech Republic \\ ${ }^{3}$ Central European Institute of Technology, CEITEC IPM, Žižkova 22, CZ-616 62 Brno, Czech Republic \\ ${ }^{4}$ Department of Chemistry, Faculty of Science, Masaryk University, Kotlářská 2, CZ-611 37 Brno, Czech Republic \\ ${ }^{5}$ Central European Institute of Technology, CEITEC MU, Masaryk University, Kamenice 5, CZ-625 00 Brno, Czech Republic
}

(Received 30 July 2020; accepted 14 September 2020; published 2 October 2020)

\begin{abstract}
To shed a new light on the complex microstructural evolution in the Ti-Al-Mo system, we employ $a b$ initio calculations to study bec-fcc structural transformations of ordered $\beta_{0}-\mathrm{TiAl}(+\mathrm{Mo})$ and disordered $\beta$ - TiAl $(+\mathrm{Mo})$ to ordered $\gamma-\mathrm{TiAl}(+\mathrm{Mo})$ and hypothetically assumed disordered $\gamma_{\mathrm{dis}}-\mathrm{TiAl}(+\mathrm{Mo})$ alloys, respectively. In particular, tetragonal (Bain's path) and trigonal transformations are combined with the concept of special quasirandom structures (SQS) and examined. Our calculations of the ordered phases show that the $\beta_{\mathrm{o}} \rightarrow$ $\gamma$ tetragonal transformation of TiAl is barrierless, i.e., proceeds spontaneously, reflecting the genuine structural instability of the $\beta_{\mathrm{o}}$ phase. Upon alloying of $\approx 7.4$ at. $\% \mathrm{Mo}$, a small barrier between $\beta_{\mathrm{o}}$ and $\gamma$-related local energy minima is formed. Yet a higher Mo content of $\approx 9$ at. $\%$ leads to an opposite-direction barrierless transformation $\gamma \rightarrow \beta_{0}$, i.e., fully stabilizing the $\beta_{\mathrm{o}}$ phase. Considering the disordered phases, the $\beta-\mathrm{Ti}_{0.5} \mathrm{Al}_{0.5-x} \mathrm{Mo}_{x}$ and $\gamma_{\mathrm{dis}}-\mathrm{Ti}_{0.5} \mathrm{Al}_{0.5-x} \mathrm{Mo}_{x}$ are energetically very close. Importantly, for all here-considered compositions up to 11 at. $\%$ of Mo, a small energy barrier separates $\beta-\mathrm{TiAl}(+\mathrm{Mo})$ and $\gamma_{\mathrm{dis}}-\mathrm{TiAl}(+\mathrm{Mo})$ energy minima. Finally, a trigonal path was studied as an alternative transformation connecting disordered $\beta$ and $\gamma_{\text {dis }}-$ TiAl phases, but it turns out that it exhibits an energy barrier over $60 \mathrm{meV} /$ at. which, in comparison to the Bain's path with $9 \mathrm{meV} /$ at. barrier, effectively disqualifies the trigonal transformation for the TiAl system.
\end{abstract}

DOI: 10.1103/PhysRevMaterials.4.103604

\section{INTRODUCTION}

Owing to their favorable mechanical properties, intermetallic $\gamma$-TiAl-based alloys are attractive structural materials for high-temperature applications in the aircraft and automotive industry. As these alloys combine a low density of roughly $4 \mathrm{~g} / \mathrm{cm}^{3}$ with a high specific yield strength and Young's modulus even at high temperatures, they are often considered as light-weight alternatives to nickel-base superalloys [1-6]. However, an obstacle for wider applications of the facecentered cubic (fcc) $\gamma$-TiAl-based alloys is their limited hot workability [7]. This shortcoming can be partially eliminated by taking advantage of the phase diversity in the Ti-Al system, which can be further extended by alloying.

For example, a stabilization of the disordered bodycentered cubic (bcc) $\beta$-TiAl [A2, space group No. 229 $(\operatorname{Im} \overline{3} m)]$ phase at elevated temperatures by adding $\mathrm{Mo}, \mathrm{Nb}$, $\mathrm{Ta}$, or $\mathrm{V}$, allows to significantly improve the processing characteristics of the $\gamma$-TiAl-based alloys [8-11]. Depending on the exact chemical composition, the $\beta$ phase may even be stabilized at room temperature as ordered $\beta_{\mathrm{o}}-\mathrm{TiAl}[\mathrm{B} 2$, space group No. $229(P m \overline{3} m)]$ next to the equilibrium phases $\gamma$-TiAl $\left[\mathrm{L}_{0}\right.$, space group No. $\left.123(P 4 / m m m)\right]$ and $\alpha_{2}-\mathrm{Ti}_{3} \mathrm{Al}\left[\mathrm{D}_{19}\right.$, space group No. $\left.194\left(P 6_{3} / m m c\right)\right][11,12]$. The alloys contain-

\footnotetext{
*neda.abdoshahi@unileoben.ac.at

†david.holec@unileoben.ac.at
}

ing the disordered $\beta$ and a minimum volume fraction of the ordered $\beta_{\mathrm{o}}$ phase have recently proven to satisfy requirements for industrial applications and thus have raised a lot of attention. They can be realized by alloying TiAl with Mo, a strong $\beta$-stabilizing element $[13,14]$, leading to the $\beta$ and $\beta_{\text {o }}$ phases appearing in the equilibrium phase diagram. Consequently, a ternary Ti-Al-Mo system turns out to be a good model system for basic research of application-relevant materials.

The facts that (i) the existence of the disordered $\beta$ and the ordered $\beta_{\mathrm{o}}$ sensitively depends on the (local) chemical composition and (ii) the TiAl-based alloys have a rather complicated multiphase nature raise a number of fundamental questions related to the stability of individual phases and transformations between them. For example, the nonexistence of the stoichiometric $\beta_{\mathrm{O}}$-TiAl phase in the binary Ti-Al system has been linked to its mechanical and vibrational instability [15]. The calculated elastic constants, $C_{11}=77 \mathrm{GPa}$, $C_{12}=129 \mathrm{GPa}$ and $C_{44}=68 \mathrm{GPa}$, do not fulfill Born-Huang stability criteria for cubic systems, by failing the necessary condition $C_{11}-C_{12}>0$. Moreover, the structural connection between the $\beta_{\mathrm{o}}$ and $\gamma$ phases involving a tetragonal deformation known as the Bain's path was shown to be barrierless and hence has been suggested to lead to a spontaneous $\beta_{\mathrm{o}} \rightarrow \gamma$ transformation [15].

This intriguing interplay between the structural disorder, Mo alloying, and phase stability motivated us to study the bcc-fcc transformations in both ordered and disordered phases of TiAl with and without Mo atoms. We explored two 

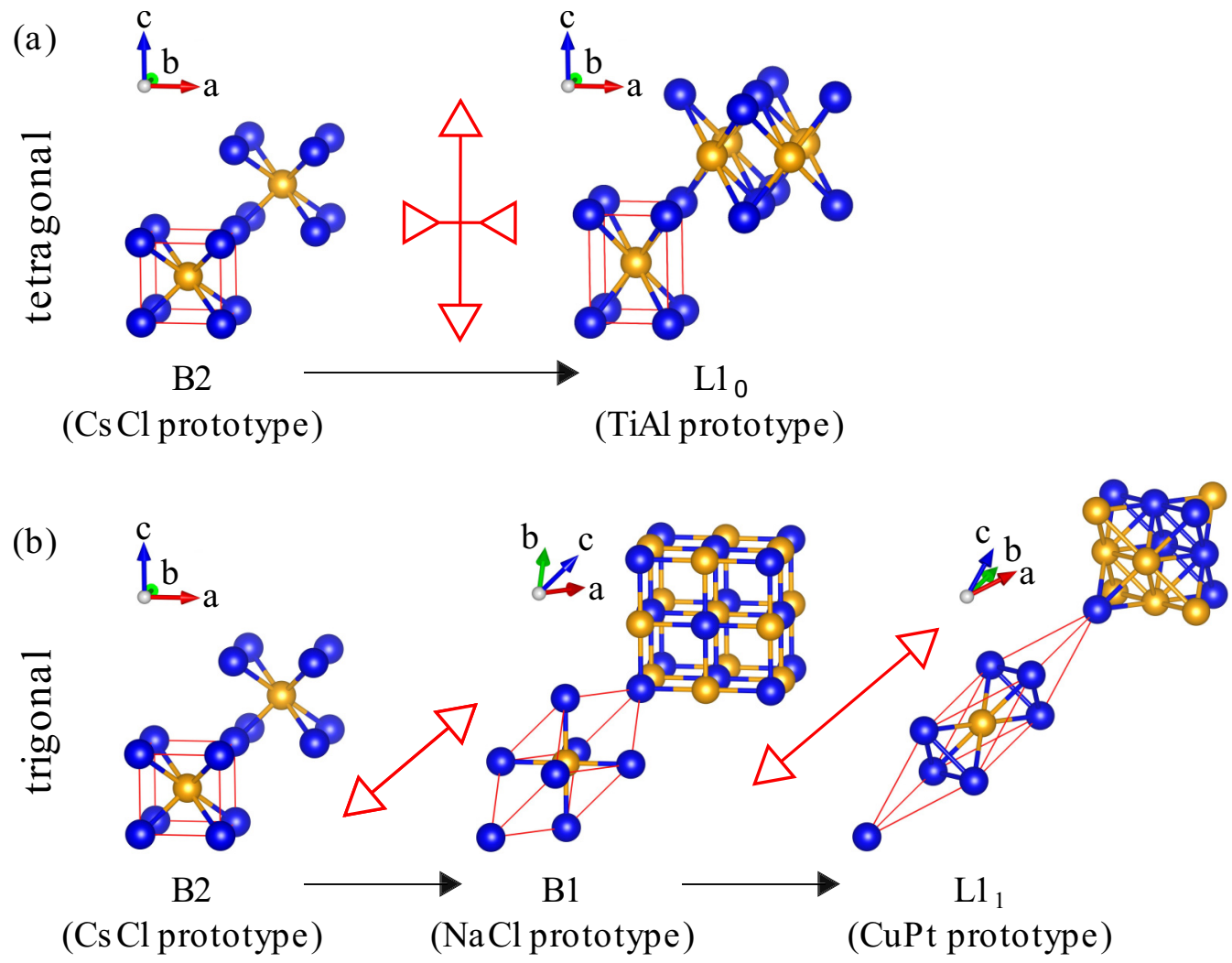

FIG. 1. Visualization of the considered structural transformations: (a) tetragonal (Bain's) path connecting the $\beta_{\mathrm{o}}$ (B2) and $\gamma$ (L1 $1_{0}$ ) structures and (b) trigonal path connecting the $\beta_{\mathrm{o}}(\mathrm{B} 2)$ and $\mathrm{L} 1_{1}$ structures via an intermediate $\mathrm{B} 1$ structure. In the case of disordered systems, all sites are occupied by both $\mathrm{Ti}$ and $\mathrm{Al}$ (and $\mathrm{Mo}$ ) atoms with an equal probability reflecting the desired composition, and both paths connect the bcc and the fcc structures, whereby the trigonal path contains an intermediate simple cubic structure. The red arrows schematically show the applied deformation (tetragonal or trigonal).

possible bcc-fcc structural connections, namely the tetragonal Bain's path [16-18] and a trigonal path [17-24] which were innovatively combined with the concept of special quasirandom structures to model the structural disorder (see Sec. II below). Afterwards we focus on clarifying the impact of Mo alloying on the energetics of transformations connecting the ordered phases $\left(\beta_{\mathrm{o}} \leftrightarrow \gamma\right)$ as well as disordered ones $(\beta \leftrightarrow$ $\left.\gamma_{\text {dis }}\right)$. At the end we discuss our theoretical predictions in the light of available experimental data.

\section{METHODOLOGY}

\section{A. Bcc-to-fec structural connections}

To investigate the transformation barriers, it is necessary to define structural connection(s) of the initial and final phases of interest. When a body-centered cubic phase is represented by a conventional cubic cell, a simple tetragonal deformation leads to a face-centered cubic structure represented by a body centered tetragonal cell (bct) [Fig. 1(a)]. This is known as the Bain's transformation path [16-19,25-29]. Let $a$ and $c$ be the lattice constants of the bcc or bct structure; $a=c=a_{\mathrm{bcc}}$ in the former state, i.e., $c / a=1$. Now consider an fcc conventional cell with a cubic lattice parameter $a_{\mathrm{fcc}}$; the lattice parameters of the corresponding bct cell are $a=a_{\mathrm{fcc}} \sqrt{2} / 2$ and $c=a_{\mathrm{fcc}}$, i.e., $c / a=\sqrt{2}$. Using a transformation coordinate $\Delta$, where $\Delta=0$ corresponds to the bcc structure whereas
$\Delta=1$ represents the bct (fcc) structure, the lattice parameters of the bct cell change as

$$
\begin{gathered}
a(\Delta)=(1-\Delta) a_{\mathrm{bcc}}+\Delta \frac{\sqrt{2}}{2} a_{\mathrm{fcc}}, \\
c(\Delta)=(1-\Delta) a_{\mathrm{bcc}}+\Delta \cdot a_{\mathrm{fcc}} .
\end{gathered}
$$

During this transformation, the $(100)_{\mathrm{bcc}}$ plane converts to the $(110)_{\mathrm{fcc}}$ while the $(001)_{\mathrm{bcc}}$ remains the $(001)_{\mathrm{fcc}}$ plane. We note that some authors (see, e.g., Refs. [19,22,24,29]) directly used $c / a$ as the transformation coordinate changing from $c / a=1(\Delta=0)$ to $c / a=\sqrt{2}(\Delta=1)$. Another important point is that the lattice parameters change linearly along the transformation path. Consequently, volume is neither linearly changing with $\Delta$ nor it is constant as, e.g., in Ref. [17]. Nonetheless, in the particular case of the Bain's path in the $\mathrm{TiAl}+$ Mo system, these different definitions result in negligible changes of the energy landscape since the specific volumes of the $\beta_{\mathrm{o}}$-TiAl $\left(V=16.19 \AA^{3} /\right.$ at. $)$ and the $\gamma$-TiAl $\left(V=16.25 \AA^{3} /\right.$ at. $)$ phases are almost identical.

When applying the Bain's transformation path to the ordered $\gamma$-TiAl phase which is a slightly tetragonally deformed fcc structure, the lattice parameters $a_{\mathrm{fcc}}$ in Eqs. (1) and (2) are replaced by $a_{\gamma}$ and $c_{\gamma}$, respectively. The Bain's transformation path is schematically shown in Fig. 1(a) for the ordered structures $\beta_{\mathrm{o}} \leftrightarrow \gamma$ and the two chemical species Ti and $\mathrm{Al}$ are shown as spheres with different colors (blue and orange, 
respectively). In the case of transformations between binary disordered structures, $\beta \leftrightarrow \gamma_{\text {dis }}$, all lattice sites are equivalent, occupied randomly by $\mathrm{Ti}$ and $\mathrm{Al}$ atoms with the same probability (all atoms in the figure would have the same color).

The trigonal path is another type of a bcc-to-fcc transformation (see, e.g., Refs. [16-28,30,31]). As shown in Fig. 1(b), it is defined by an extension along the [111] direction of the bcc structure. The ordered cubic-symmetry structures forming an equimolar binary compound are $\mathrm{B} 2(\mathrm{CsCl}$ prototype), $\mathrm{B} 1$
( $\mathrm{NaCl}$ prototype), and $\mathrm{L}_{1}(\mathrm{CuPt}$ prototype). They can be conveniently described by a suitable transformation coordinate $\Delta$ with values $\Delta=0$ for the bcc (B2 in the case of ordered binary compound) phase, $\Delta=\frac{1}{3}$ for the simple cubic (sc) (B1) phase and $\Delta=1$ for the fcc $\left(\mathrm{L}_{1}\right)$ phase. The lattice vectors' matrix defining the unit cell is given as a function of the transformation coordinate $\Delta$, following the graphical representation in Fig. 1(b) by also considering the possible specific volume change along the transformation path, as

$$
\mathbf{M}(\Delta)= \begin{cases}a(\Delta)\left(\begin{array}{lll}
1 & \epsilon & 0 \\
0 & 1 & \epsilon \\
\epsilon & 0 & 1
\end{array}\right), \epsilon=3 \Delta, & \text { if } 0 \leqslant \Delta \leqslant \frac{1}{3}, \\
a(\Delta)\left(\begin{array}{ccc}
1 & 1-\epsilon & \epsilon \\
\epsilon & 1 & 1-\epsilon \\
1-\epsilon & \epsilon & 1
\end{array}\right), \epsilon=\frac{3}{4}\left(\Delta-\frac{1}{3}\right), & \text { if } \frac{1}{3} \leqslant \Delta \leqslant 1 .\end{cases}
$$

The unit cell volume $V=\operatorname{det} \mathbf{M}$ is linearly interpolated between the high-symmetry structures along the trigonal transformation path as

$$
V(\Delta)= \begin{cases}(1-3 \Delta) V_{\mathrm{bcc}}+3 \Delta \cdot V_{\mathrm{sc}}, & \text { if } 0 \leqslant \Delta \leqslant \frac{1}{3}, \\ 3 \Delta \cdot V_{\mathrm{sc}}+\frac{3 \Delta-1}{2} V_{\mathrm{fcc}}, & \text { if } \frac{1}{3} \leqslant \Delta \leqslant 1,\end{cases}
$$

where $V_{\mathrm{bcc}}, V_{\mathrm{sc}}$, and $V_{\mathrm{fcc}}$ are equilibrium volumes of bcc (B2), sc (B1), and fcc $\left(\mathrm{L}_{1}\right)$ structures per two atoms, respectively.

We note that our definitions of the trigonal transformation path allow for volume changes between the $\beta_{\mathrm{o}}$ and $\mathrm{L} 1_{1}$, and $\beta$ and $\gamma_{\text {dis }}$ phases, respectively. In contrast to our description, previous literature focusing on trigonal path with constant volume used $c / a$ as a transformation coordinate [17,18]. There, $c$ is the length of the [111] vector in a nonorthogonal coordination system defined by rows of the matrix $\mathbf{M}$ in Eq. (3), whereas $a=|[11 \overline{2}]| / \sqrt{2}$. Knowing additionally the relation between $V=\operatorname{det} \mathbf{M}$ and $\Delta$ [in the present case given by Eq. (4)], one can directly relate $c / a$ to $\Delta$. This is shown in Fig. 2.

\section{B. Computational details}

We performed calculations based on density functional theory (DFT) as implemented in the Vienna Ab initio Simulation Package (VASP) [32] using Projector Augmented-Wave method (PAW) potentials $[33,34]$. The exchange and correlation potential was described at the generalized gradient approximation (GGA) level using the Perdew-Burke-Ernzerhof (GGA-PBE) parametrization [35]. The valence configurations of used (pseudo)potentials of Ti, Al, and Mo were [Ar] $3 d^{2} 4 s^{2}$, $[\mathrm{Ne}] 3 p^{1}$, and $[\mathrm{Kr}] 4 d^{5} 5 s^{1}$, respectively. A convergence threshold of $10^{-6} \mathrm{eV}$ was used for the total energy of the electronic self-consistency, whereas structural relaxations were stopped for the total energy changes smaller than $10^{-4} \mathrm{eV}$. The wave functions were expanded in plane waves up to a kinetic energy cutoff of $500 \mathrm{eV}$. The spacing of $k$-points used for sampling the irreducible part of the first Brillouin zone was $\approx 0.1 \AA^{-1}$. This translates to $19 \times 19 \times 19 k$-point mesh for the reciprocal lattice corresponding to the conventional cubic cell of the ordered $\beta_{\mathrm{o}}$-TiAl. The calculations were converged with respect to the number of $k$-points and plane wave cutoff energy to reach the total energy accuracy of $1 \mathrm{meV} /$ at. or better.

When computing properties of disordered states (with and without Mo atoms), two types of supercells were used. One type contained 54 atoms as a $3 \times 3 \times 3$ multiple of the 2 -atom $\mathrm{bcc} / \mathrm{bct}$ conventional cell of the ordered $\beta_{\mathrm{o}}$-TiAl. The second type contained 32 atoms as a $2 \times 2 \times 2$ multiple of a 4 -atom conventional $\mathrm{fcc} / \gamma$-TiAl cell. The atomic distributions inside the supercells were generated following the special quasirandom structures (SQSs) concept [36] with optimized pair correlation functions up to the seventh coordination shell [37] using an in-house software tool [38].

The energy changes are discussed in terms of formation energy $E_{\mathrm{f}}$ defined as

$$
\begin{aligned}
E_{\mathrm{f}}= & E_{0}\left(\mathrm{Ti}_{m} \mathrm{Al}_{n} \mathrm{Mo}_{l}\right)-\frac{1}{m+n+l}\left[m E_{0}(\mathrm{hcp}-\mathrm{Ti})\right. \\
& \left.+n E_{0}(\mathrm{fcc}-\mathrm{Al})+l E_{0}(\mathrm{bcc}-\mathrm{Mo})\right],
\end{aligned}
$$

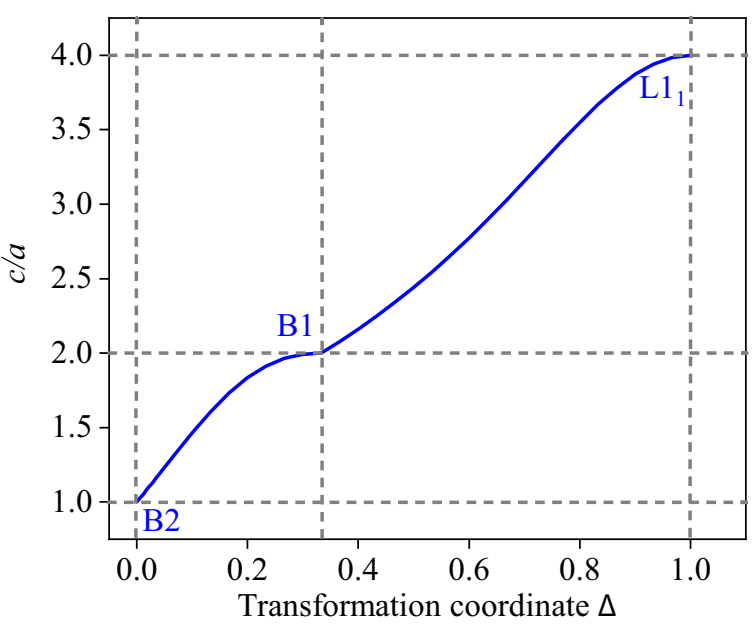

FIG. 2. Dependence of $c / a=\sqrt{2}|[111]| /|[11 \overline{2}]|$ on the transformation coordinate $\Delta$ for the trigonal transformation defined by Eqs. (3) and (4). $|[\cdot]|$ means length of a vector [ [ ] in a nonorthogonal coordination system which is defined by the rows of matrix $\mathbf{M}$ in Eq. (3). 
where $E_{0}\left(\mathrm{Ti}_{m} \mathrm{Al}_{n} \mathrm{Mo}_{l}\right)$ is the total energy (per atom) of a supercell with $m, n$, and $l$ atoms of $\mathrm{Ti}, \mathrm{Al}$, and Mo, respectively, representing the studied phases. $E_{0}(\mathrm{hcp}-\mathrm{Ti}), E_{0}(\mathrm{fcc}-\mathrm{Al})$, and $E_{0}$ (bcc-Mo) are the total energies (per atom) of $\mathrm{Ti}, \mathrm{Al}$, and Mo elements in their standard (reference) states, respectively.

The effect of temperature on the formation energies is included in the framework of the Debye model as formulated in the recent publications $[39,40]$. This provides a formula for approximating the vibrational free energy as a function of temperature and material equilibrium volume and elastic properties at $0 \mathrm{~K}$. The elastic constants, namely bulk modulus $B$ and Poisson's ratio $v$, were obtained from the full tensor of elastic constants calculated with the stress-strain method [41], which were subsequently projected on an isotropic elasticity [42]. The thus-obtained values of the bulk modulus were cross-checked against those obtained from the BirchMurnaghan equation of state [43] for structural optimizations with fixed cell shape.

The site or sublattice preference is crucial for constructing reasonable models of the ordered phases containing Mo. Jiang [44] reported weak (compositionally dependent) preference for Mo in $\gamma$-TiAl. This means that Mo prefers to sit on $\mathrm{Al}$ (Ti) sublattice in the case of Ti-rich (Al-rich) compositions (with respect to stoichiometric $\gamma$-TiAl). On the contrary, Holec et al. [45] concluded that Mo exhibits a strong Al sublattice preference in the case of the $\beta_{\mathrm{o}}$ phase, i.e., that Mo sits on the $\mathrm{Al}$ sublattice for both, Ti-rich and Al-rich off-stoichiometries (in the later case antisite defects would be generated together with alloying Mo into $\beta_{\mathrm{o}}$-TiAl). More details on the terminology and methodology of site preference in ordered systems can be found in Refs. [44,45] and references therein. In this work, we focus on Ti-rich ternary $\mathrm{Ti}_{0.5} \mathrm{Al}_{0.5-x} \mathrm{Mo}_{x}$ compositions, in agreement with experimentally investigated alloys [46,47], and hence Mo occupies Al sublattice sites in the case of ordered phases.

\section{RESULTS}

\section{A. Ground-state properties}

The structural instability of some of the studied phases emerges already when searching for the minimum-energy configurations. In particular, there is a significant dependency of a computational model of the disordered $\beta$-TiAl phase on constraints applied during its structural relaxation. When the simulation box shape is fixed to a cubic one, the structural relaxation results in large, seemingly disordered, local atomic displacements, see Fig. 3(a), while a full structural relaxation leads to significant change of the cell shape [see Fig. 3(b)].

For example, one local structural motif (lemon trapezium) in the first case changes to a clear rectangle with $a=2.886 \AA$ and $c=4.061 \AA$ upon a full relaxation. These values are notably similar to the $\gamma$-TiAl lattice parameters expressed in a tetragonal body-centered cell: $a=2.826 \AA$ and $c=4.062 \AA$ [11]. On the other hand, the blue area in Fig. 3(a) resembling a sheared square $(a=3.167 \AA, c=3.417 \AA)$, changes to a general nonrectangular shape in Fig. 3(b). Consequently, also the disordered structures spontaneously transform similar to $\beta_{\mathrm{o}} \rightarrow \gamma$, however, the transformation $\beta \rightarrow \gamma_{\mathrm{dis}}$ is to be expected to take place only locally.
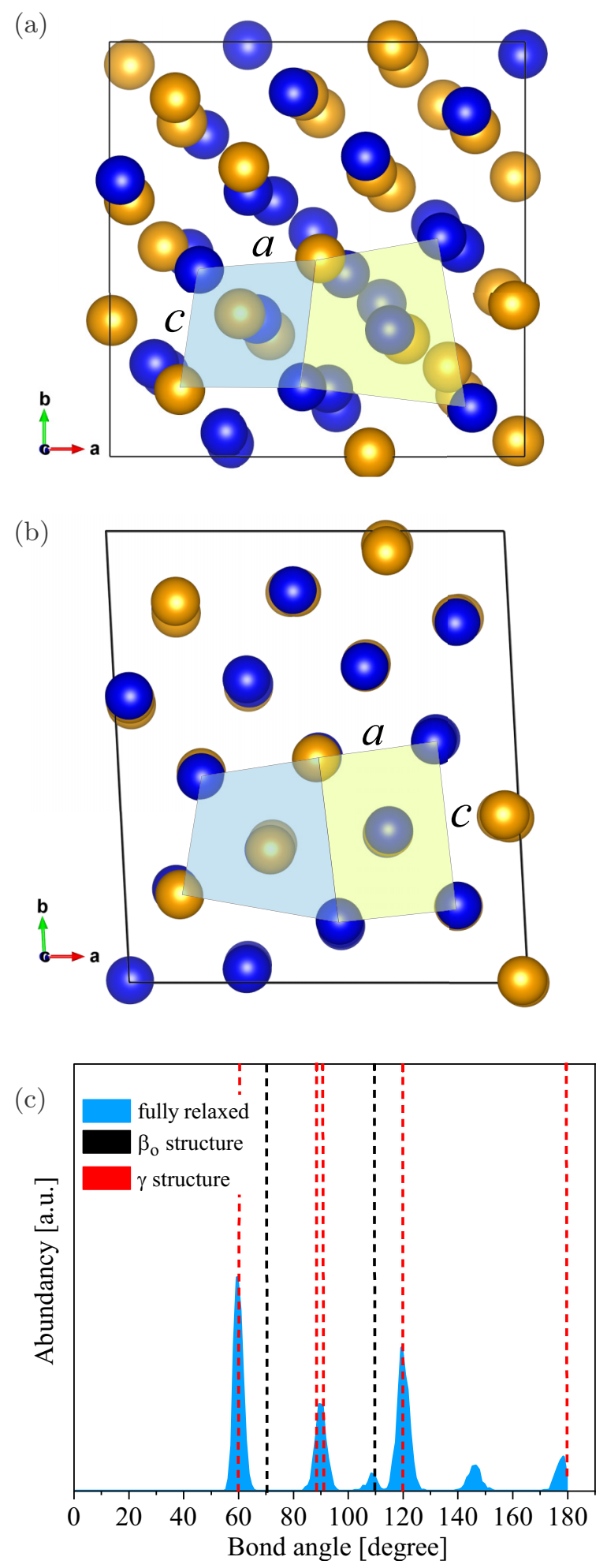

FIG. 3. Schematic visualization of the minimum-energy configuration of the disordered $\beta$-TiAl phase when (a) preserving the overall cubic cell shape and (b) when fully relaxing the supercell. The blue and green motifs result in significantly different shapes depending on the relaxation mode. (c) Histogram of bond angles of the fully relaxed structure. 


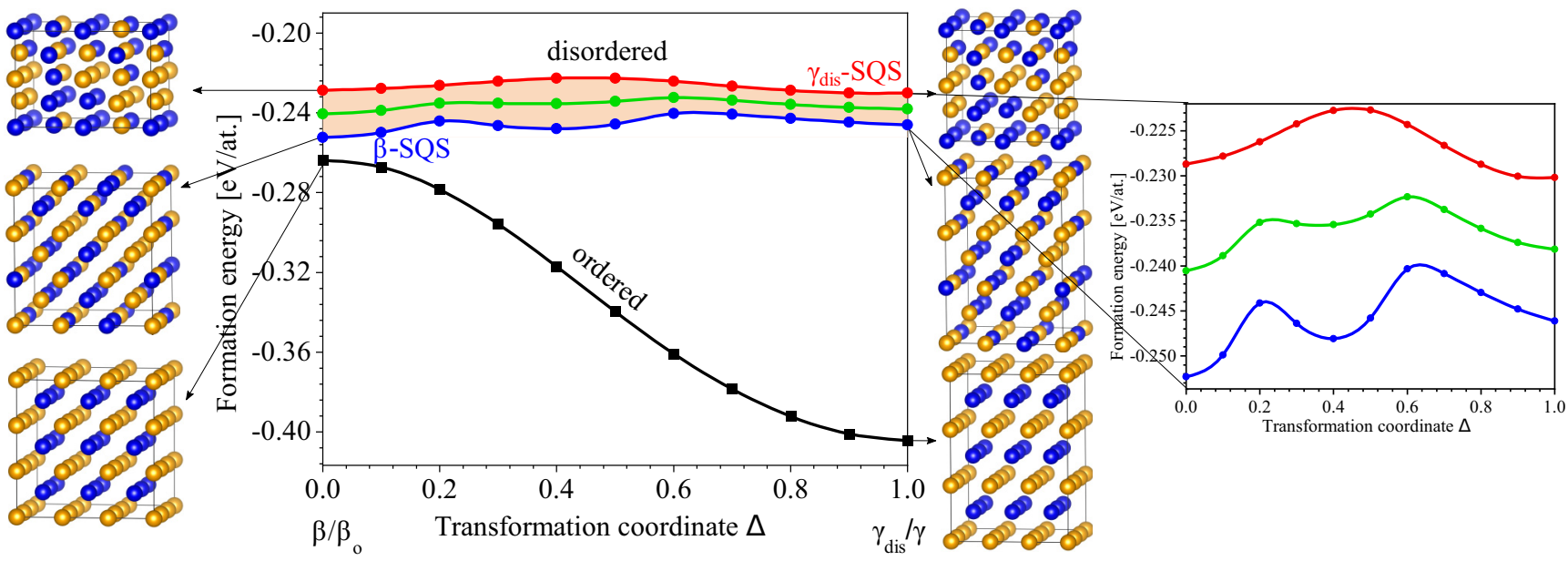

FIG. 4. The computed changes of the formation energy along the Bain's tetragonal transformation path in the case of ordered phases $\beta_{\mathrm{o}} \rightarrow \gamma$ as well as disordered ones $\beta \rightarrow \gamma_{\text {dis. }}$. The inset magnifies the energy changes associated with the transformation of disordered phases with the red and blue curves corresponding to bcc-optimized and fcc-optimized SQSs, respectively (see them visualized along the vertical axes). The green curve in the middle between the red and blue curves represents the average of the energies along the $\beta \leftrightarrow \gamma_{\text {dis }}$ transformation. The black curve indicates the formation energy changes related to the $\beta_{\mathrm{o}} \leftrightarrow \gamma$ transformation of the ordered phases.

The structural relaxations in the disordered $\beta$-TiAl structure, can also be visualized as a histogram of bond angles [Fig. 3(c)] corresponding to the fully relaxed structure from Fig. 3(b). When compared to the structural fingerprints of ideal ordered $\beta_{\mathrm{o}}$-TiAl phase (black) and ordered $\gamma$-TiAl structure (red), a significant overlap with the $\gamma$ structure is apparent. However, some bond angles around $110^{\circ}$ still resemble the initial (unrelaxed) disordered $\beta$ (ordered $\beta_{\mathrm{o}}$ ) structure, while others around $145^{\circ}$ do not agree with either of the two fingerprints. This only further highlights the complexity and richness of the structural relaxations demonstrating the structural instability of the bcc-based TiAl (see also our previous results in Ref. [15]). In what follows, we used models with desired (fixed) cell shape and fully relaxed internal degrees of freedom (atomic positions).

\section{B. Tetragonal Bain's path for the stoichiometric TiAl phases}

After determining and analyzing the ground-state structures, we proceed with the investigation of configurations along the Bain's tetragonal deformation path $\beta_{\mathrm{o}} \leftrightarrow \gamma$ (Fig. 4). The lattice parameters were set according to Eqs. (1) and (2). The atomic positions remain fixed by the crystal symmetry of the ordered $\beta_{\mathrm{o}}$ and $\gamma$ TiAl phases. The energy extrema correspond to $\Delta=0\left(\beta_{0}\right.$-TiAl, maximum $)$ and $\Delta=1(\gamma-$ $\mathrm{TiAl}$, minimum). The second of these is consistent with the fact that the $\gamma$ phase is the ground state for stoichiometric $\mathrm{Ti}_{0.5} \mathrm{Al}_{0.5}$. Both of the above-mentioned extrema can be understood in terms of so-called symmetry-dictated extrema. Note that while our transformations are not volume-conserving, the energies change by less than $0.1 \mathrm{meV} /$ at. when the volume is kept constant, well below the accuracy of our calculations, and the arguments of Craievich et al. [25] can be applied in our case as well. Namely, those authors showed that some energy extrema on energy profiles along the constant-volume transformation paths are dictated by the symmetry. Most of the structures along a transformation path between two highersymmetry structures, here between $\mathrm{B} 2$ and $\mathrm{L} 1_{0}$ at the Bain's path, have a symmetry that is lower than cubic. At those points of the transformation path where the symmetry of the structure is higher, the derivative of the total energy with respect to the parameter describing the path must be zero. These are the so-called symmetry-dictated extrema. However, other extrema may occur that are not dictated by the symmetry and reflect properties of the specific material. For more details on different types of extrema we refer the reader to Refs. [16-19,22,24,25,29].

The $\beta_{\mathrm{o}} \rightarrow \gamma$ Bain's path between ordered phases exhibits no transformation barrier (i.e., it is a barrierless or spontaneous transformation) and a net gain in energy is equal to

$$
\Delta E\left(\beta_{\mathrm{o}} \rightarrow \gamma\right)=E\left(\beta_{\mathrm{o}}\right)-E(\gamma)=140 \mathrm{meV} / \text { at } .
$$

This value is in good agreement with previous studies, for instance, Paidar et al. [17] reported the energy difference along the Bain's path for the $\beta_{\mathrm{o}} \rightarrow \gamma$ case of ordered phases equal to about $140 \mathrm{meV} /$ at. while Šb et al. [18] obtained about $150 \mathrm{meV} /$ at.

Next we investigated the same transformation also for the disordered variants of the ordered $\beta_{\mathrm{o}}$ and $\gamma$ phases, i.e., $\beta \leftrightarrow \gamma_{\text {dis }}$. In particular, the blue curve (bottom of the shaded area) in Fig. 4 corresponds to a bcc-optimized SQS structure ( $\beta$-SQS), which is transformed to $\gamma_{\mathrm{dis}}-\mathrm{TiAl}$ (fcc). This means that the short-range order parameters were optimized for the bcc lattice (e.g., the first coordination shell contains eight nearest neighbors) and obviously the Warren-Cowley SRO parameters may no longer be optimal after the transformation to the fcc $\gamma_{\text {dis }}-$ TiAl phase with 12 nearest neighbors. Taking the fcc structure instead and optimizing its SRO parameters we obtained another supercell (this time with 32 atoms, i.e., $2 \times 2 \times 2$ conventional 4 -atomic fcc cell). This supercell $\left(\gamma_{\mathrm{dis}}-\mathrm{SQS}\right)$ for $\gamma_{\mathrm{dis}}-\mathrm{TiAl}$ is tetragonally transformed into the $\beta$-TiAl yielding the data on the red curve. Therefore, while both blue and red curves are describing the same process, the difference between them may be interpreted as the SQS accuracy limit of our calculations (given also the chosen supercell size). Consequently, a simple average (green curve) of these 
curves is used as a representative for the $\beta \leftrightarrow \gamma_{\text {dis }}$ transformation path of disordered phases with the color-shaded area being an estimate of the accuracy (Fig. 4). The inset clearly shows that small barriers between the two phases occurs for both SQS types, although the actual energy barrier(s) and energy landscapes slightly differ. In summary, our calculations confirm the spontaneous character of the diffusionless transformation $\beta_{\mathrm{o}} \rightarrow \gamma$ of the ordered phases driven by a mechanical instability (long-wavelength phonons) in the $\beta_{0^{-}}$ TiAl phase. This driving force is significantly reduced (the red curve Fig. 4), or even completely reversed (the blue curve in Fig. 4) in the case of the disordered $\beta$-TiAl phase and its hypothetical, chemically disordered counterpart $\gamma_{\text {dis }}-\mathrm{TiAl}$. Moreover, a (small) energy barrier exists in the disordered case preventing the transformation to proceed spontaneously. Therefore, these results confirm our previous findings that the chemical disorder relatively stabilizes the bcc-based $\beta$-TiAl [15].

\section{Trigonal transformation in the stoichiometric TiAl}

In this section we explore another structural transformation connecting the bcc-like $\beta_{\mathrm{o}}$ and $\beta$ on the one hand and the fcc-like states $\gamma$ and $\gamma_{\text {dis }}$ on the other hand via the trigonal transformation path described in detail in Sec. II A. The transformation barriers are shown in Fig. 5.

The B2 lattice parameter $\left(a_{\mathrm{B} 2}=3.187 \AA\right)$ changes to $a_{\mathrm{B} 1}=2 a_{\mathrm{sc}}=5.292 \AA$ and further to $a_{\mathrm{L} 1_{1}}=2 a_{\mathrm{fcc}}=8.132 \AA$. These changes correspond to specific volumes of $V_{\mathrm{B} 2}=$ $16.19 \AA^{3} /$ at., $V_{\mathrm{B} 1}=18.52 \AA^{3} /$ at. and $V_{\mathrm{L}_{1}}=16.81 \AA^{3} /$ at., respectively. For a trigonal path with the specific volume interpolated between the high-symmetry structures, a barrier of $503 \mathrm{meV} /$ at. (see purple curve with star symbols) is obtained. This value is smaller than the previously reported values of $520 \mathrm{meV} /$ at. [17] and $540 \mathrm{meV} /$ at. [18]. This stems from the fact that the past reports assumed constant volume along the transformation path. To test this hypothesis, we calculated the energies along the trigonal path with keeping the volume constant (and fixed to $V_{\mathrm{B} 2}$ [see the orange curve in Fig. 5(a)], yielding a barrier of $596 \mathrm{meV} /$ at.). Importantly, the maximum along the trigonal path is obtained in either case for the $\mathrm{B} 1$ structure, while the $\mathrm{B} 2\left(\beta_{\mathrm{o}}\right)$ and $\mathrm{L} 1_{1}$ structures represent local minima.

The final $\mathrm{L} 1_{1}$ structure represents a metastable phase. Its energy is higher than that of the $\beta_{0}$ structure by $76 \mathrm{meV} /$ at. and by $217 \mathrm{meV} /$ at. higher than the $\gamma$-TiAl $\left(\mathrm{L}_{0}\right.$ structure), the ground state of TiAl. Similar values of $61 \mathrm{meV} /$ at. and $71 \mathrm{meV} /$ at. were reported for $E\left(\mathrm{~L} 1_{1}-\mathrm{B} 2\right)$ by the authors or Refs. $[17,18]$, respectively, while the same authors obtained of $215 \mathrm{meV} /$ at. and $212 \mathrm{meV} /$ at. for $E\left(\mathrm{~L}_{1}-\mathrm{L}_{0}\right)$. The $\mathrm{B} 1$ structure represents a local maximum/saddle point and the energy can be further decreased towards the $\mathrm{L} 1_{1}$ structure for increasing the trigonal distortion. Although this state is (at least thermodynamically) stable, it is not the here investigated $\gamma$-TiAl phase and hence will not be further discussed. Moreover, the barriers are significant and the trigonal transformation is energetically less convenient than the spontaneously proceeding tetragonal path. Consequently, the trigonal transformation path cannot connect the ordered $\beta_{0^{-}}$ TiAl and $\gamma$-TiAl phases (which has the $\mathrm{L} 1_{0}$ lattice), although
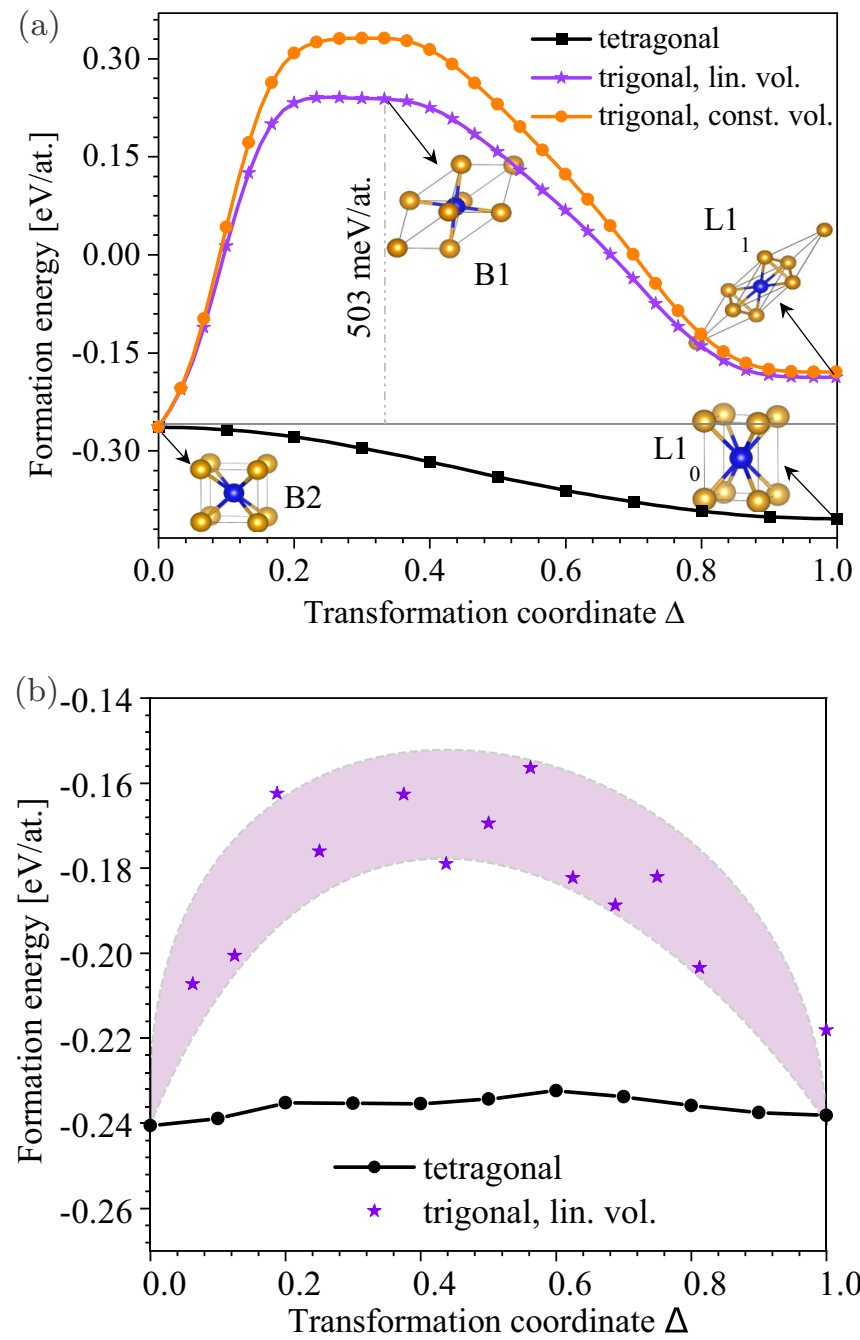

FIG. 5. The formation energy along the tetragonal (Bain's) and the trigonal transformation paths for (a) ordered $\beta_{\mathrm{o}} \rightarrow \gamma / \mathrm{L} 1_{1}$ and (b) disordered $\beta \rightarrow \gamma_{\text {dis }}$ TiAl.

it offers a possible structural transformation path connecting the bcc and fcc disordered states investigated below.

The situation is rather different in the case of disordered states due to the lack of a higher symmetry [Fig. 5(b)]. The intermediate state corresponding to a simple cubic structure turned out very difficult to structurally optimize, and therefore we focused on linearly interpolating specific volumes between the values corresponding to the bcc $\beta-\operatorname{TiAl}\left(a_{\mathrm{bcc}}=3.207 \AA\right.$, $V_{\mathrm{bcc}}=16.49 \AA^{3} /$ at. $)$ and fcc $\gamma_{\mathrm{dis}}-\mathrm{TiAl}\left(a_{\mathrm{fcc}}=4.05 \AA, V_{\mathrm{fcc}}=\right.$ $16.61 \AA^{3} /$ at.) phases. The structural complexity manifest itself by the rather scattered data for different $\Delta$ along the trigonal path. Therefore, the energies are represented by a shaded region with upper and lower envelopes for the reader's convenience [Fig. 5(b)] . Although the trigonal barrier is an order of magnitude lower than that for the ordered phases, it is still significantly higher than for the tetragonal Bain's path: $\approx 60-90 \mathrm{meV} /$ at. for the trigonal deformation as compared to $\approx 10 \mathrm{meV} /$ at. for the tetragonal Bain's path. Therefore, the trigonal transformation is also in the case of disordered phases rather unlikely to be the actual transformation mechanism as the tetragonal path exhibits significantly smaller (to 


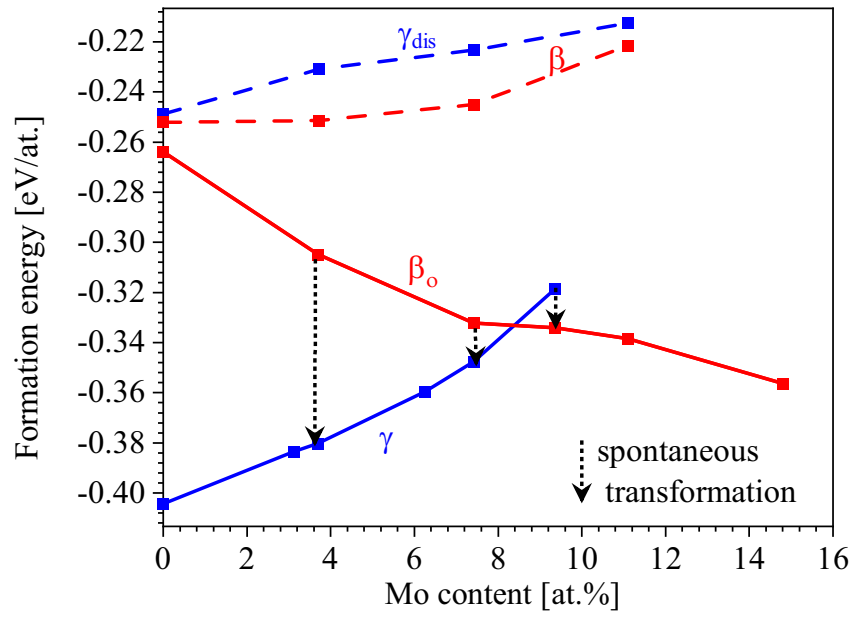

FIG. 6. The calculated formation energy of the ordered $\beta_{0}$ and $\gamma$ as well as the disordered $\beta$ and $\gamma_{\text {dis }}$-TiAl as a function of the Mo content. The dashed black arrows show spontaneous transformation as obtained during the structural relaxation at $0 \mathrm{~K}$.

negligible) barriers. We can thus conclude that the (spontaneous) structural transformation between both ordered $\beta_{\mathrm{o}} \rightarrow$ $\gamma$ and disordered states $\beta \rightarrow \gamma_{\text {dis }}$ is more likely to proceed via the tetragonal Bain's path instead of the trigonal transformation.

\section{Impact of Mo on the $\beta_{0} \leftrightarrow \gamma$ and $\beta \leftrightarrow \gamma_{\text {dis }}$ transformations in TiAl}

As has been mentioned in the Introduction, the pure $\beta_{0}$ TiAl phase does not exist - it exhibits mechanical and dynamical instability leading to a spontaneous barrierless transformation of the $\beta_{0}$ phase into the stable $\gamma$-TiAl [15]. Since Mo can be added to TiAl in order to stabilize the $\beta_{\mathrm{o}} / \beta$ phases [11], we next study the impact of Mo on the transformation energy landscape of both ordered $\beta_{\mathrm{o}} \leftrightarrow \gamma$ and disordered $\beta \leftrightarrow \gamma_{\text {dis }}$ states.

The computed energies of formation are shown in Fig. 6. Focusing now on the ordered phases, for a low Mo content, the $\gamma$ phase is more stable than the $\beta_{\mathrm{o}}$ variant (corresponding to the driving force for the spontaneous transformation at $x_{\text {Mo }}=0$ ), but with increasing Mo content the energy difference between these two phases decreases and finally at $\approx 8$ at. $\%$ Mo the $\beta_{\mathrm{o}}$-TiAl + Mo phase becomes more stable than the $\gamma$-TiAl + Mo variant. The intersection point of the energy of $\beta_{\mathrm{o}}$ and $\gamma$ phases denotes the ranges of stability of the single phases, which can co-exist with identical composition exactly at Mo content corresponding to this cross-over.

In contrast to the ordered phases discussed above, the disordered phases exhibit a preference for the bcc structure over the fcc one for all considered Mo compositions (Fig. 6). Although the $\beta$ phase is slightly preferred over the $\gamma$ phase, the reader should recall the energy differences presented in Fig. 4 for different cells (different sizes, different relaxations). The energy difference between $\beta$ and $\gamma_{\text {dis }}$ remains similar to that between different SQSs and supercell sizes (Fig. 4). In fact, local structural motifs resembling a hexagonal structure

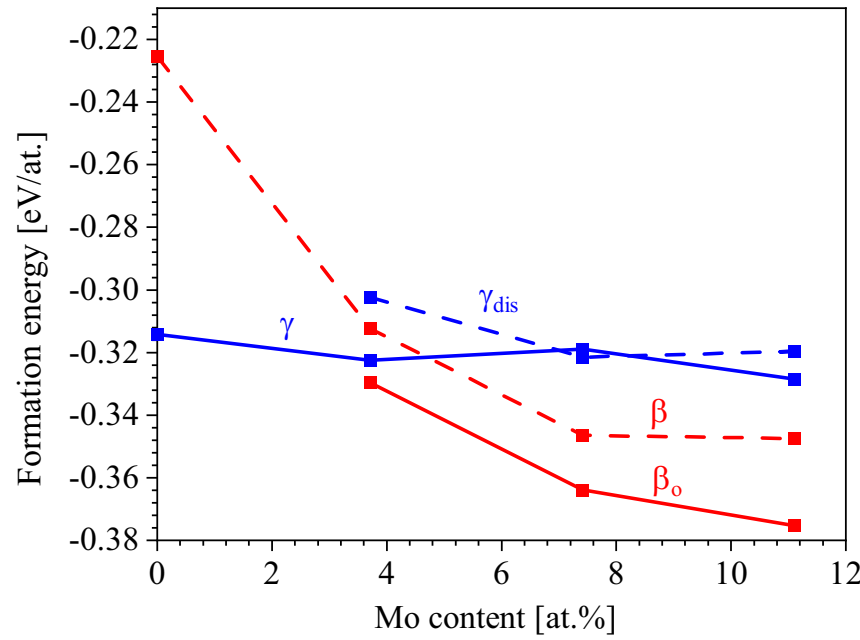

FIG. 7. Formation energies of $\beta_{\mathrm{o}} / \beta$ and $\gamma / \gamma_{\text {dis }}$ phases as function of Mo content $\left(\mathrm{Ti}_{0.5} \mathrm{Al}_{0.5-x} \mathrm{Mo}_{x}\right)$ at $1000 \mathrm{~K}$ calculated with the Debye model.

can be detected after the full relaxation which could indicate a relaxation-induced transformation towards a hexagonal $\alpha$ based phase. A martensitic transformation of the $\beta$-TiAl to a hexagonal phase has been recently experimentally reported $[47,48]$ and hence such relaxation may possibly be related to this diffusionless transformation.

Employing the Debye model, we calculated vibrational contribution to the free energy of all four intermetallic phases, as well as of elemental $\mathrm{Al}, \mathrm{Ti}$, and Mo metals, and used these values to calculate formation energy at finite temperature. The total energy of the binary/ternary systems [the first term on the right-hand side of Eq. (5)] was further corrected for the configurational entropy on the Al sublattice (ordered systems) or in the whole simulation cell (disordered systems). Figure 7 shows the resulting $E_{f}$ as functions of Mo content corresponding to $1000 \mathrm{~K}$. The most important effect of the temperature is the stabilization of the $\beta_{\mathrm{o}} / \beta$ phases with respect to the $\gamma$ phase. We note that the pure $\beta_{\mathrm{o}}$-TiAl phase is mechanically unstable, and hence the Debye model (requiring knowledge of elastic constants) is not applicable. It is also worth mentioning that $\gamma / \gamma_{\text {dis }}$ and $\beta_{\mathrm{o}} / \beta$ phases become energetically very close. This indicates that the systems are approaching the order/disorder transition at temperatures somewhat higher than $1000 \mathrm{~K}$. The Debye model as used here is quite a crude approximation (it considers elastically isotropic material, further no explicit treatment of phonons, no anharmonicity, etc. is included), and thus the present results should be regarded only as demonstrating temperaturerelated trends rather than accurately predicting ordering temperatures.

Figure 8(a) shows the transformation energy landscape along the tetragonal path as a function of the Mo content for the ordered phases. While the $\gamma$ structure is clearly preferred for the considered Mo content of 0 and 3.7 at. $\%$ with no energy barrier between the $\beta_{\mathrm{o}}$ and the $\gamma$ phase, the situation is completely opposite for the 9.3 at. $\%$ Mo when the $\beta_{0}$-phase is energetically preferred over the $\gamma$ phase without any transformation barrier. An interesting case occurs for 7.4 at.\% Mo 
(a)

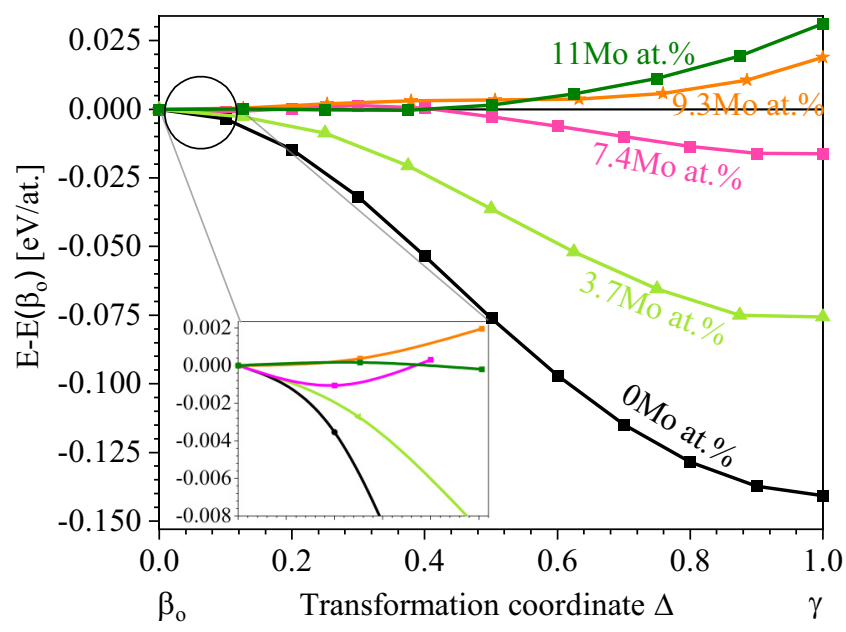

(b)

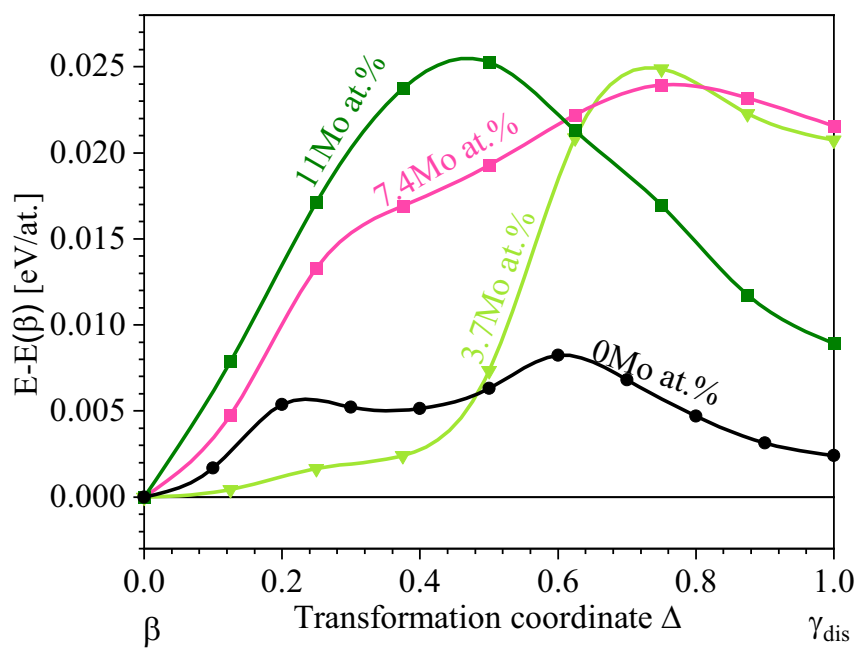

FIG. 8. The computed energy profile along the tetragonal (Bain's) path for ordered $\mathrm{Ti}_{0.5} \mathrm{Al}_{0.5-x} \mathrm{Mo}_{x}$ phase connecting (a) the ordered $\mathrm{B} 2-\beta_{0}-\mathrm{TiAl}+\mathrm{Mo}(\Delta=0)$ and $\mathrm{L}_{0}-\gamma-\mathrm{TiAl}+\mathrm{Mo}(\Delta=1)$ structures and (b) disordered bcc- $\beta$-TiAl $+\operatorname{Mo}(\Delta=0)$ and fcc- $\gamma_{\text {dis }}-$ $\mathrm{TiAl}+\operatorname{Mo}(\Delta=1)$ structures. The energies are shown relative to the energy of the $\beta_{\mathrm{o}} / \beta$ phase with a respective Mo content.

when the $\gamma$ phase exhibits more negative $E_{f}$ than the $\beta_{\mathrm{o}}$ phase (by $\approx 18 \mathrm{meV} /$ at.) but, importantly, the Bain's path shows a small energy barrier (of about $\approx 1 \mathrm{meV} /$ at.). Despite its very small height, the barrier opens the possibility of stabilizing both phases, i.e., the $\beta_{\mathrm{o}}$ phase would become metastable in this particular case.

Figure 8(b) shows energy changes along the $\beta \rightarrow \gamma_{\text {dis }}$ Bain's transformation path of several studied $\mathrm{Ti}_{0.5} \mathrm{Al}_{0.5-x} \mathrm{Mo}_{x}$ compositions. Unlike in the case of the ordered phases [Fig. 8(a)], the disordered phases do not exhibits any clear trend as a function of Mo content. In agreement with formation energies (Fig. 6), the $\gamma_{\text {dis }}$ phases have higher energy than the $\beta$ phases. Also, in contrast to the ordered phases, $\beta \rightarrow \gamma_{\text {dis }}$ always exhibit barriers (although in many cases very small) separating the $\beta$ and $\gamma_{\text {dis }}$ phases. Consequently, the chemical disorder effectively prevents any spontaneous phase transformations as predicted for the case of ordered $\beta_{\mathrm{o}}$ and $\gamma$ phases.

\section{DISCUSSION}

\section{A. Structural peculiarities of the TiAl(+Mo) system}

Our results summarized in the previous section clearly show that the $\operatorname{TiAl}(+\mathrm{Mo})$ system exhibits a number of rather unusual properties. For example, when inspecting the energetics of both ordered and disordered TiAl phases transformed along the Bain's path (see Fig. 4), it is striking how small energy changes are induced by this structural transformation in the disordered $\beta \rightarrow \gamma_{\text {dis }}$ case.

An inset at the right-hand side of Fig. 4 visualizes energy barriers of a few $\mathrm{meV} /$ atom that could be easily overcome. Consequently, the disordered $\beta$-TiAl is expected to be quite deformable even in an elastic manner. Despite the disordered $\beta$-TiAl is experimentally not observed, the above-mentioned deformability can be expected for other phases and/or compositions. For example, the composition of the ordered $\beta_{0}$-TiAl stabilized by 9.3 at.\% Mo is close to those found in experimental samples and the energy barriers along a major part of the Bain's tetragonal path are also only a few $\mathrm{meV} /$ atom [see Fig. 8(a)]. Therefore, it is worthwhile to analyze the structural behavior of different phases at an atomic scale along the studied tetragonal path in detail.

Starting with the above-mentioned disordered $\beta$-TiAl phase and the very small energy changes induced in it by the tetragonal transformation, there are a few facts that should be mentioned.

First, the volume-conserving Bain's transformation is characterized by only very small changes in the first nearestneighbor distances. Therefore, the $1 \mathrm{NN}$ bonds are re-oriented in space rather then significantly shortened. The reorientation process is energetically less costly presumably due to the dominant metallic nature of the bonds.

Second, both $\mathrm{Ti}$ and $\mathrm{Al}$ were reported to exhibit rather small energy differences between the bcc and fcc structures along the Bain's path (about $100 \mathrm{meV} /$ at. and $40 \mathrm{meV} / \mathrm{at}$. for $\mathrm{Ti}$ and $\mathrm{Al}$, respectively, see, e.g., Craievich et al. [49]). These energy differences are smaller than $E\left(\beta_{0}-\gamma\right)=$ $140 \mathrm{meV} /$ at. for TiAl. Moreover, none of these two elements is stable in the bcc structure in their ground state and they both spontaneously transform from the bcc structure (symmetry-dictated energy maximum) to the fcc structure (symmetry-dictated energy minimum) in a barrierless manner.

As much as these two reasons may intuitively justify the $\beta_{\mathrm{o}} \rightarrow \gamma$ behavior, they cannot completely explain the computed results along the whole tetragonal path as the same arguments hold equally well for the disordered $\beta$-TiAl, which has the same composition and its energy changes along the tetragonal Bain's transformation path are an order of magnitude smaller (about $10 \mathrm{meV} /$ at.).

The major difference between the disordered $\beta$-TiAl and its ordered counterpart is related to its internal atomic configuration and it is not only the fact that $\beta$-TiAl has the atoms distributed in a disordered manner. As already discussed above [and shown in Fig. 3(a)], the $\beta$-TiAl is internally significantly distorted and the atoms exhibit a tendency to locally resemble the structure of $\gamma$-TiAl. We found that the same significant internal distortions exist in structures encountered along a major part of the Bain's path (Fig. 9), in particular for structures from $\Delta=0$ up to $\Delta=0.6$. We note that similar 

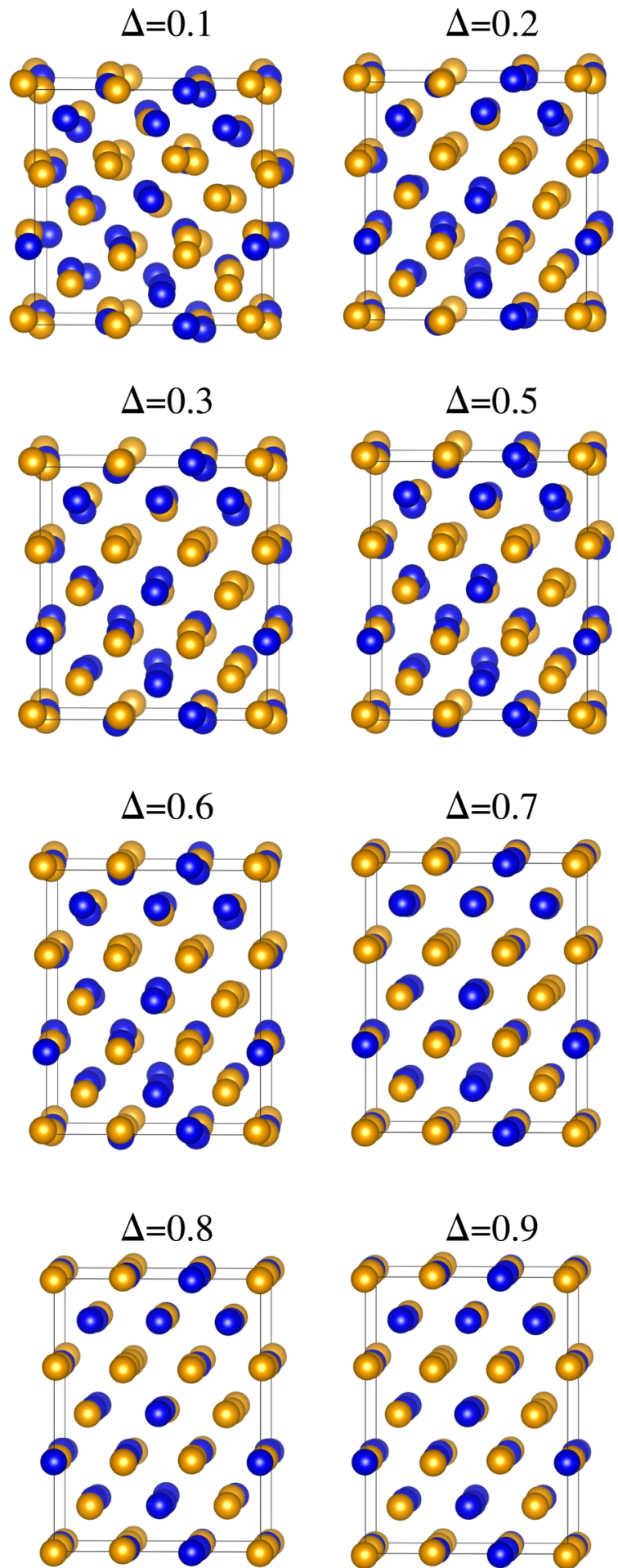

FIG. 9. Visualization of relaxed internal atomic positions in 54atom SQS supercell as obtained from minimization of the total energy with respect to the atomic coordinates for selected states along the Bain's path.

behavior is obtained also for the low-Mo containing $\mathrm{TiAl}+$ Mo systems.

To analyze distortions of the internal atomic structures quantitatively, we studied radial distribution functions in the structures encountered along the tetragonal transformation path (Fig. 10). Using a comparison to the ordered $\beta_{0}$-TiAl, the differences in the atomic distributions are clearly visible. In particular, the second coordination $(2 \mathrm{NN})$ sphere with six atoms in the ordered $\beta_{0}$-TiAl (at $\Delta=0$ ) splits upon transformation, and four of its atoms continuously move along the path towards the first coordination (1NN) shell in the $\gamma$ phase $(\Delta=1)$, thereby increasing the number in the first coordination cell to 12 (not considering the small tetragonality of the $\gamma$-TiAl), see Fig. 10(a). The other two atoms move further away to join some of the atoms from the third nearest neighbor (3NN) shell (at $\Delta=0$ ) to form the $2 \mathrm{NN}$ shell in the $\gamma$ phase $(\Delta=1)$. In contrast to these well-defined coordination shells in the case of the ordered $\beta_{\mathrm{o}}$-TiAl, there is hardly any $2 \mathrm{NN}$ shell visible in the radial distribution functions of the disordered $\beta$-TiAl [Fig. 10(b) and the magnification in Fig. 10(c)]. The peak corresponding to the $1 \mathrm{NN}$ shell is significantly broadened covering the distances from $2.8 \AA$ to $3.2 \AA$. It effectively means that the environment indeed resembles that in the $\gamma$ phase already for $\Delta=0$.

Therefore, it should be emphasized that the small energy changes in the disordered TiAl along the Bain's path are not those associated with a process when a crystal structure changes from one with strictly eight atoms in the first nearest neighbor (1NN) shell to another with 12 atoms in the $1 \mathrm{NN}$ shell. It is rather so that the $\mathrm{Ti}$ and $\mathrm{Al}$ atoms locally rearrange (whenever possible) so as to have an fcc-like environment even in configurations that have the shape of the simulation box corresponding to small $\Delta$ values.

To further analyze the importance of the above-mentioned internal distortions we also computed a situation when the $\beta$ TiAl phase is chemically disordered but the atoms are located in perfect and undistorted lattice positions, e.g., bcc-like for $\Delta=0$. Computationally, it means that the atomic positions were not relaxed, i.e., the total energy was not minimized with respect to the atomic positions. It turns out that the mechanical and thermodynamic stability of such internally undistorted structures is very (even qualitatively) different from the internally distorted (relaxed) ones (Fig. 11). The energy of unrelaxed structures is significantly higher than that of their relaxed (internally distorted) counterparts (these energies are same as in Fig. 4). In particular, the energies of the unrelaxed structures with $\Delta=0$ are clearly higher than those with $\Delta=1$. The energy dependence of configurations close to the $\Delta=0$ is very flat and there is no energy barrier along the transformation path. Consequently, the undistorted structures with $\Delta=0$ have quite a different mechanical stability: it is much reduced (when compared with the internally distorted configuration with $\Delta=0$ ), it is, in fact, on the verge of instability as there is hardly any barrier for a transformation to the (internally undistorted) $\gamma$ structure with $\Delta=1$.

When discussing the unusual properties of the $\mathrm{TiAl}+\mathrm{Mo}$ system, it is worth mentioning the impact of Mo atoms on the structures that are tetragonally transformed. Regarding the internal atomic configuration of TiAl + Mo phases, the presence of Mo atoms in both ordered and disordered TiAl leads to distortions that are very similar to those in disordered binary TiAl. Employing the analysis of the RDFs along the tetragonal Bain's path again, our results are summarized for 7.4 at.\% of Mo in Fig. 12. Starting with the ordered TiAl + $\mathrm{Mo}$, the $1 \mathrm{NN}$ and $2 \mathrm{NN}$ shells merge for $\Delta=0$ into a single 

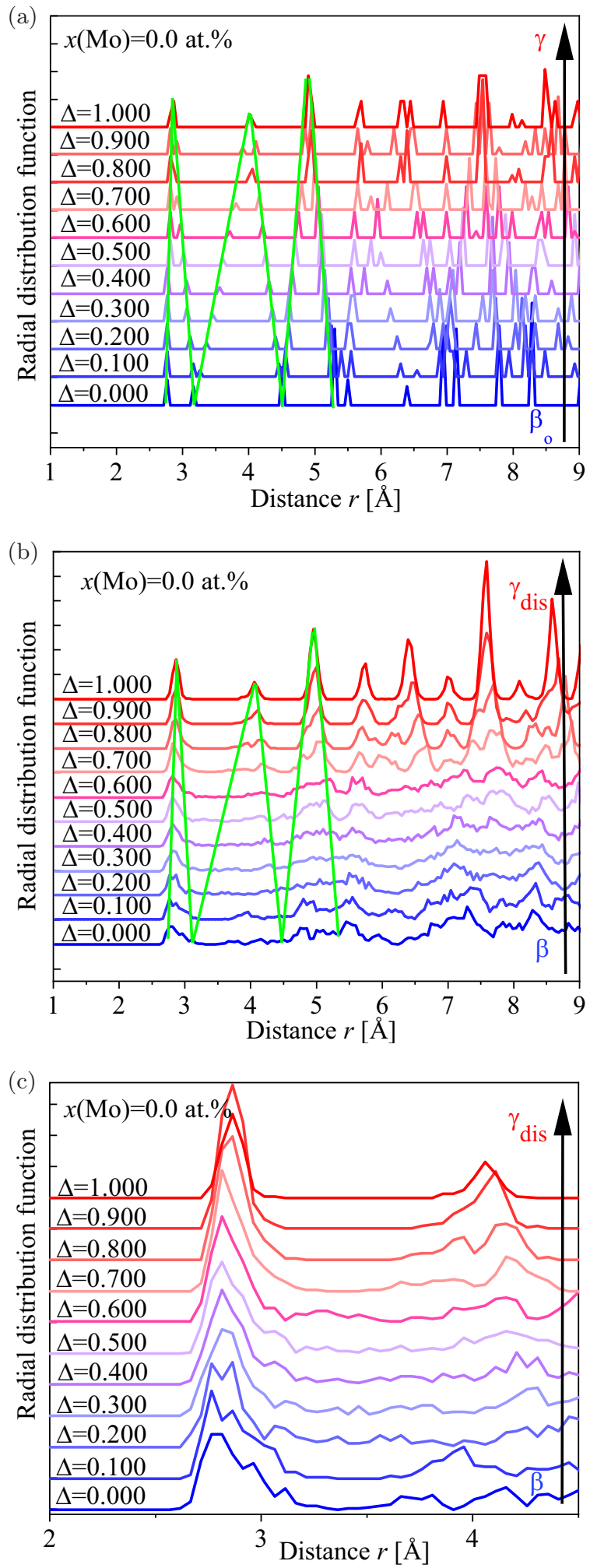

FIG. 10. Radial distribution functions for (a) the ordered $\beta_{0}$-TiAl and (b) the disordered $\beta$-TiAl phases, tetragonally transformed to the $\gamma / \gamma_{\text {dis }}$ phases. Part (c) is a magnified part of (b). The straight lines schematically indicate shifts in selected nearest-neighbor shells of atoms.

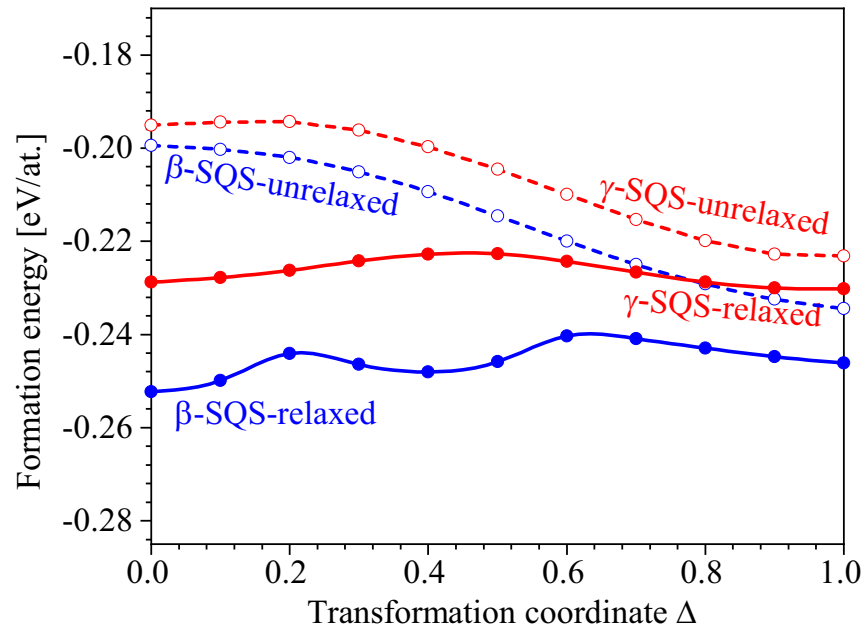

FIG. 11. Comparison of energies along the $\beta \rightarrow \gamma_{\text {dis }}$ Bain's transformation path of disordered TiAl for $\beta$-optimized (blue) and $\gamma$ optimized (red) SQSs with relaxed (full lines) and unrelaxed (dashed lines) internal positions.

broad peak [cf. Fig. 10(a)]. We interpret this as a tendency to merge the atoms from the $2 \mathrm{NN}$ with those in the $1 \mathrm{NN}$ to form locally an environment that mimics the $\gamma$ phase. This can be a crucial fact as the Mo atoms stabilize the ordered TiAl phase making it eventually stable even in the $\beta$ structure.

As far as the disordered TiAl structures with Mo additions are concerned, we exemplify the trends in the case with 7.4 at.\% Mo [see Figs. 12(b) and 12(c)]. The 2NN shell is so broadened and partly merged with both the $1 \mathrm{NN}$ shell and other shells that it is very difficult to resolve them [see the magnification in Fig. 10(c)]. This behavior resembles that in the disordered binary TiAl visualized in Figs. 10(b) and 10(c). Here it is worth mentioning that the chemical disorder and Mo additions are two mechanisms stabilizing the $\beta_{0} / \beta$ phases of TiAl + Mo. It is interesting that their combination, i.e., Mo additions into the disordered $\beta$-TiAl does not enhance the stabilization effect. On the contrary, the impact of Mo atoms on the thermodynamic stability of disordered $\beta$-TiAl + Mo is negative, i.e., Mo atoms energetically destabilize the disordered TiAl + Mo phase, as visible in the trends of the formation energy in Fig. 6. This trend is reverted with temperature (including configurational and vibrational entropy), see Fig. 7. We therefore conclude that the stabilizing effect of Mo is related to (i) replacing Ti-Al bonds with Ti-Mo (ordered $\beta_{\mathrm{o}}-\mathrm{TiAl}+\mathrm{Mo}$ ) and (ii) significant contribution to vibrational entropy at high temperatures.

\section{B. Supercell size versus SQS}

We now focus a bit closer on the SQS supercells and the fact that the energy values shown in Fig. 4 for two different SQS supercells exhibit a difference of about $20 \mathrm{meV} /$ at. We assign this apparent discrepancy to differences in the supercell shapes and/or sizes leading to differently optimized shortrange order parameters during their generation. In particular, the $\beta$-SQS is a $3 \times 3 \times 3$ supercell based on a 2 -atom bcc conventional cubic cell, thus containing 54 atoms. Consequently, the eighth coordination shell is the first one containing 

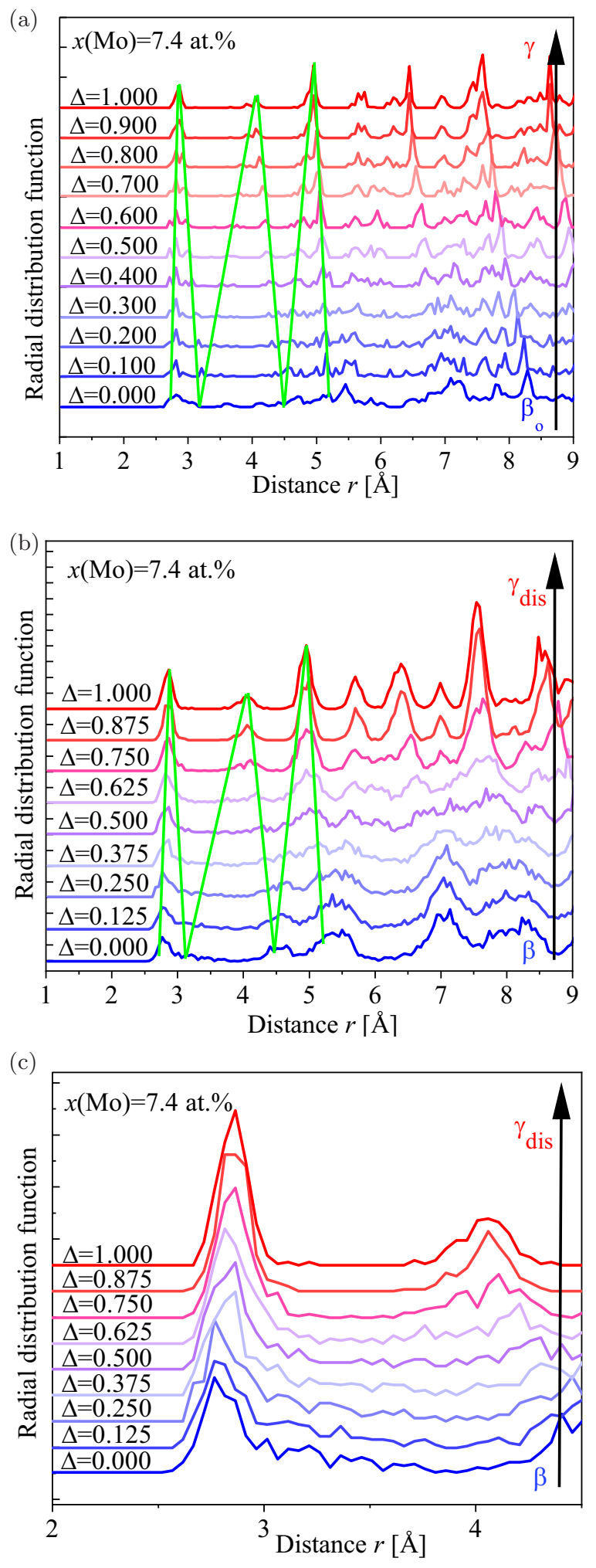

FIG. 12. Radial distribution functions for (a) the ordered $\beta_{0}$ and (b) disordered $\beta$-TiAl + Mo phases with 7.4 at.\% Mo additions. (c) is a magnified part of the RDFs from the (b). The straight green lines schematically indicate shifts in selected nearest-neighbor shells of atoms. periodic images of atoms (located in the neighboring periodically repeated supercells), making only the first seven coordination shells available for an independent optimization of the atomic disorder. On the contrary, the $\gamma_{\text {dis }}-S Q S$ is a $2 \times 2 \times 2$ supercell based on the conventional 4-atom fcc cell containing 32 atoms. Here, already the sixth coordination shell contains periodic images of atoms. Therefore, in this case, only atoms in the first five coordination shells can be independently optimized. The short-range order of atoms in a binary alloy can be conveniently described by Warren-Cowley short-range order (SRO) parameters defined as

$$
\alpha_{i}=1-\frac{N_{\mathrm{AB}}}{N M_{i} x_{\mathrm{A}} x_{\mathrm{B}}},
$$

where $N_{\mathrm{AB}}$ is the number of A-B pairs at the $i$ th coordination shell in a supercell with $N$ sites, $M_{i}$ is the coordination of each site on the $i$ th shell, and $x_{\mathrm{A}}$ and $x_{\mathrm{B}}=1-x_{\mathrm{A}}$ are compositions of atoms $\mathrm{A}$ and $\mathrm{B}$, respectively.

It turns out that our two structural models with 54 and 32 atoms have the Warren-Cowley short-range order parameters responding to the simulated tetragonal transformation very differently (see Table I). In particular, while the 54-atom cell has zero $\alpha_{1}$ and $\alpha_{3}$ parameters corresponding to the first and third shells in the bcc structure, respectively, the absolute values of the first three SRO parameters $\alpha_{1}, \alpha_{2}$, and $\alpha_{3}$ are significantly nonzero for the Bain-transformed $\beta$-SQS to the fcc structure (the overall $\alpha=0.0228$ for $\Delta=1$ ). Here we note that the objective function combines individual SRO parameters $\alpha_{i}$ (specifically, their absolute values) with arbitrary weights, here with factors $1 / i$. These decreasing weights $1 / i$ represent an intuitive expectation that the nearest interactions are the most important. However, they do not reflect any specific chemistry of the studied system. The identified complexity related to the SQS description of strong longrange interactions present in the $\mathrm{Ti}-\mathrm{Al}$ intermetallic system, in particular for the small cells, is in agreement with a previous study on the supercell-size effect on accuracy of elastic constants in the Ti-Al systems [50]. It was shown that the 32-atomic supercell is to be expected to have an error of around $1 \%$, while increasing the size to 108 atoms, the error drops to approximately half of that [50].

\section{Spontaneous transformation as a function of the Mo content}

Let us discuss in detail our results obtained for the formation energies given in Fig. 6. In particular, it should be noted that the points in Fig. 6 represent the calculated energies based on different supercells with different sizes with the Mo atoms distributed according to the SQS concept. The $\beta_{\mathrm{O}}$-based SQS supercells contain 54 atoms while the $\gamma$-based SQS supercells contain 32 atoms. As a consequence of different sizes, the Mo consentrations in the 32-atom and 54-atom supercells are slightly different (one Mo atom corresponds to 1.85 at. $\%$ in the $\beta_{\mathrm{o}}$-based supercells and 3.12 at. $\%$ in the $\gamma$-based supercell). Thus, for example, while the datapoint at $x_{\mathrm{Mo}}=3.12$ at. $\%$ on the $\gamma$ dataset in Fig. 6 corresponds to one Mo atom in the $\gamma$-based supercell, the data point at $x_{\mathrm{Mo}}=$ 3.70 at. $\%$ is a fully structurally relaxed $\beta_{\mathrm{o}}$ phase with two Mo atoms in the supercell (i.e., spontaneously transformed $\beta$-SQS once also cell shape optimization was allowed). 
TABLE I. The short-range order parameters of the 54-atom ( $\beta$-SQS) and 32-atom $\left(\gamma_{\mathrm{dis}}-\mathrm{SQS}\right)$ supercells used in our calculations. The parameter $\Delta$ is the transformation coordinate with $\Delta=0$ corresponding to the bcc structure and $\Delta=1$ to the fcc structure. $\alpha_{i}$ is the WarrenCowley short-range order parameter for the $i$ th coordination shell and $\alpha$ is their weighted sum used as an objective function during the SQS generation [38].

\begin{tabular}{|c|c|c|c|c|c|c|c|c|c|}
\hline & $\Delta$ & $\alpha$ & $\alpha_{1}$ & $\alpha_{2}$ & $\alpha_{3}$ & $\alpha_{4}$ & $\alpha_{5}$ & $\alpha_{6}$ & $\alpha_{7}$ \\
\hline \multirow[t]{2}{*}{$\beta$-SQS } & 0 & 0.0088 & -0.0000 & -0.0062 & 0.0000 & -0.0185 & 0.0000 & -0.0021 & 0.0053 \\
\hline & 1 & 0.0228 & -0.0123 & 0.0062 & -0.0123 & 0.0000 & -0.0074 & -0.0062 & 0.0053 \\
\hline \multirow{2}{*}{$\gamma_{\mathrm{dis}}-\mathrm{SQS}$} & 0 & 0.0200 & 0.0000 & 0.0000 & 0.0000 & 0.0000 & -0.1000 & & \\
\hline & 1 & 0.0208 & 0.0000 & 0.0000 & 0.0000 & -0.0833 & 0.0000 & & \\
\hline
\end{tabular}

These differences also mean that special attention must be paid when comparing spontaneous transformations in Fig. 6 (see the vertical black dashed arrows) with the tetragonal Bain's transformations in Fig. 8(a). The comparison is straightforward for, e.g., a spontaneous transformation of $\beta_{\mathrm{o}} \rightarrow \gamma$ shown in Fig. 6 for 3.7 at.\% Mo, which is fully in agreement with the transformation energetics depicted in Fig. 8(a). A similar situation is in the case of 7.4 at. $\%$ (4 Mo atoms in the $\beta_{0}$ phase supercell), although the energy difference, i.e., the transformation driving force, is much smaller. Importantly, no $\beta_{\mathrm{o}} \rightarrow \gamma$ transformation occurs for 11.1 at. $\%$ (6 Mo atoms in the $\beta_{\mathrm{o}}$ supercell). On the contrary, the $\beta_{\mathrm{o}}$-TiAl + Mo datapoint with $x_{\mathrm{Mo}}=9.4$ at. $\%$ is actually a spontaneously transformed $\gamma$ phase (with three Mo atoms in the $\gamma$ supercell). In summary, since the structural relaxations (at $0 \mathrm{~K}$ ) are sufficient to induce phase transformations, the Born-Oppenheimer energy landscape for the tetragonal $\beta_{\mathrm{o}} \leftrightarrow$ $\gamma$ structural connection is likely to contain negligible-to-no energy barriers, in agreement with results from the previous section [Fig. 8(a)]. Therefore, the $\gamma$ and $\beta_{\mathrm{o}}$ phase can co-exist only when the local Mo content in each of them is different (up to $\approx 7$ at.\% in the $\gamma$-TiAl the less Mo, the more stable), otherwise in the case of $\beta_{\mathrm{o}}$-TiAl for yet higher Mo content.

\section{Experimental evidence}

To support the results of our calculations, let us now compare them to available experimental data. During the previous decades, phase equilibria and phase transformations in the ternary Ti-Al-Mo system have been investigated by a number of different research groups and discussed, e.g., in Refs. [12-14,51-57], to name a few. Yet only some experimental studies have explicitly addressed the phase transformations between $\beta$ (or $\beta_{\mathrm{o}}$ ) and $\gamma$ phases in Mocontaining alloys $[3,4,46,52,52]$. Singh and Banerjee [52] analyzed solidification structures of various Ti-(44-50)Al-(26)Mo (at.\%) alloys. A comparison of the measured chemical compositions, which prevail in these microstructures close to thermodynamic equilibrium, reveals large differences in the local Mo content in the $\beta / \beta_{\mathrm{o}}$ and $\gamma$ phases. For example, in the Ti-50Al-6Mo (at.\%) alloy, the primary $\beta$ phase (present at room temperature as ordered $\beta_{\mathrm{o}}$ ) exhibits a Mo content of 13.0 at. $\%$, whereas only 3.2 at.\% Mo are present in the interdendritic $\gamma$ phase. In Ti-50Al-2Mo (at.\%), there are 11.1 at. $\%$ Mo in $\beta_{\mathrm{o}}$ and only 1.2 at.\% Mo in $\gamma$, while peritectic $\alpha$ (present at room temperature as ordered $\alpha_{2}$ ) features in both microstructures as a third phase. These chemical compositions, which were determined by means of electron probe microanalysis, support our conclusion drawn in the previous results section, namely that the co-existence of $\gamma$ and $\beta_{\mathrm{o}}$ must be connected with differences in the local Mo content. Furthermore, they confirm our prediction that the $\beta_{\mathrm{o}}$ phase is more stable than $\gamma$ for Mo contents of 8 at.\% and higher, while for lower Mo contents a spontaneous transformation to $\gamma$ takes place.

At this point it is important to note that qualitative arguments of this kind can only be deployed if experimental conditions close to thermodynamic equilibrium apply, and the experiments were conducted at sufficiently low temperatures. In specimens that were quenched from high temperatures, also Mo contents lower than $\approx 8$ at. $\%$ can be observed in the $\beta_{0}$ phase. For example, Singh and Banerjee [52] observed Mo contents as low as 2.2 at. $\%$ and 2.9 at.\% after quenching a Ti44Al-2Mo (at.\%) alloy from $1673 \mathrm{~K}$ and $1573 \mathrm{~K}$, respectively. These microstructures are usually not stable and transform upon heating as they approach thermodynamic equilibrium. In a recent paper, Musi et al. [47] compared quenched specimens of a Ti-44Al-3Mo (at.\%) alloy to hot-isostatically pressed conditions of the same material and found (by means of electron dispersive spectroscopy in a transmission electron microscope) that the chemical composition of the $\beta_{\mathrm{o}}$ phase increased from a Mo content of 4.1 at.\% to 7.7 at.\% during annealing at $1473 \mathrm{~K}$ and slow cooling. In contrast to this, the newly formed $\gamma$ phase finally exhibited a Mo content of 1.5 at.\%, which is again in good qualitative agreement with our predictions.

Recently, the partitioning of Mo between the phases $\beta_{0}$ and $\gamma$ has also been studied experimentally in the course of an in situ heating experiment at a synchrotron radiation source [46]. For this experiment, a Ti-44Al-7Mo (at.\%) alloy was selected. According to the equilibrium phase diagram $[55,58], \beta_{\mathrm{o}}$ and $\gamma$ are the only stable phases in this alloy below roughly $1473 \mathrm{~K}$. Consequently, no other phases have to be considered during the observation of the $\beta \rightarrow \beta_{\mathrm{o}}+\gamma$ phase transformation, which occurs when the quenched and, thus, supersaturated single-phase $\beta_{\mathrm{o}}$ microstructure decomposes upon heating. Starting from the solid solution, in which $\beta$ exhibits the nominal chemical composition of the alloy, $\gamma$ particles of a reduced Mo content nucleate and grow. After heating to $953 \mathrm{~K}$ at a rate of $10 \mathrm{~K} / \mathrm{min}$, the $\gamma$ precipitates were found to have a Mo content of 3.9 at.\%. As only 8 to $10 \%$ of the matrix had transformed to $\gamma$ at this point, atom probe tomography indicated that the Mo content of the $\beta_{\mathrm{o}}$ matrix had increased only marginally. Long-term annealing of this material at $1273 \mathrm{~K}$, though, has been shown to finally yield Mo contents of 16.3 at.\% in the $\beta_{\mathrm{o}}$ and 2.0 at.\% in the 
$\gamma$ phase [4]. This behavior is again very much in agreement with our calculations. As can be deduced from Fig. 6, the overall energy of the system is minimized by the observed phase separation, during which almost pure $\gamma$ phase and $\beta_{\mathrm{o}}$ at a significantly higher Mo content are obtained. Furthermore, in accordance with our results, this transformation was found to take place without the formation of any metastable phase of different crystallographic structure.

Based on our calculations, we can even go one step further. The convex shape of the curve related to the $\beta_{\mathrm{o}}$ phase in Fig. 6 may be taken as an argument supporting the suggestions proposed in Ref. [46], namely that in the investigated Mo range, the $\beta_{\mathrm{o}}$ phase does not display any tendency towards spinodal decomposition, but that the phase separation is governed rather by a nucleation and growth process. This research question was raised, as the conditions pertaining to classic nucleation, e.g., that the $\gamma$ nuclei already show their final chemical composition, were found to be not ideally fulfilled due to the complexity of the proposed heat treatments. In this regard, our ab initio calculations successfully address a critical issue, which is, in this material system, not easily accessible by means of experimental techniques.

\section{CONCLUSION}

In this paper we used $a b$ initio calculations to study the energetic landscape of structural connections between the bccbased ordered $\beta_{\mathrm{o}}$ and disordered $\beta$ phases, on one hand, and fcc-based ordered $\gamma$ and disordered $\gamma_{\text {dis }} \mathrm{TiAl}+$ Mo phases on the other hand. In the case of the stoichiometric TiAl system, the ordered phase is predicted to transform spontaneously from the bcc $\beta_{\mathrm{o}}$ to the tetragonally distorted fcc $\gamma$ phase. Unlike that, the disorder stabilises the bcc- $\beta$ phase and raises a small barrier between the bcc and the fcc phases. In addition to the tetragonal deformation path (Bain's path), the trigonal deformation path is also investigated. This path, however, transforms the ordered TiAl into a different structural type and exhibits a large energy barrier of $\approx 500 \mathrm{meV} /$ at. in the ordered case, effectively ruling such structural connection out. A small barrier is predicted also for the transformation between disordered states. The impact of Mo, a known stabilizer of the $\beta_{\mathrm{o}} / \beta$ phase, was finally considered as well. It turns out that the bcc phase becomes energetically preferred over the $\gamma$ phase for Mo content of $\approx 7.4$ at.\% and higher. Around this critical Mo content, a small energy barrier of $\approx 1 \mathrm{meV} /$ at. is predicted. For all other compositions, the spontaneous (barrierless) nature of the Bain's path connecting the $\beta_{\mathrm{o}}$ and $\gamma$ phases is predicted to prevail. Unlike that, the Bain's path connecting the disordered bcc $\beta$ and fcc $\gamma_{\text {dis }}$ phases exhibits a barrier for all Mo concentrations considered here, effectively making it possible for both phases to co-exist. Finally, the instability of the $\beta$ - $\mathrm{TiAl}(+\mathrm{Mo})$ phase was linked to individual atomic environments, exhibiting a strong tendency to locally resemble the tetragonally distorted structure of the $\gamma_{\mathrm{dis}}-\mathrm{TiAl}(+\mathrm{Mo})$ phase.

\section{ACKNOWLEDGMENTS}

This research was funded by the Austrian Science Fund (FWF) Project No. P29731-N36. Additional resources were provided by the Ministry of Education, Youth, and Sports of the Czech Republic under the Project CEITEC 2020, LQ1601 (M.Š, M.F.) and the Academy of Sciences of the Czech Republic from its institutional support [Institutional Project No. RVO:68081723 (M.Š, M.F.)]. The computational results presented were achieved, in part, using the Vienna Scientific Cluster (VSC). Additional computational resources were made available by the Ministry of Education, Youth, and Sports of the Czech Republic under the Project IT4Innovations National Supercomputer Center (project e-Infrastructure CZ-LM2018140) within the program Projects of Large Research, Development and Innovations Infrastructures and partly also via the CESNET (Project No. LM2015042) and CERIT-Scientific Cloud (Project No. LM2015085). Parts of Figs. 1, 3 to 5, and 9 were visualized using the VESTA package [59-61].
[1] D. Dimiduk, Mater. Sci. Eng. A 263, 281 (1999).

[2] F. Appel, J. Paul, and M. Oehring, Gamma Titanium Aluminide Alloys: Science and Technology (Wiley, New York, 2011).

[3] M. Engstler, S. Mayer, C. Pauly, H. Clemens, and F. Mücklich, Adv. Eng. Mater. 15, 1125 (2013).

[4] B. Rashkova, K. Spiradek-Hahn, M. Brabetz, Z. Zhang, T. Schöberl, H. Clemens, and S. Mayer, Int. J. Mater. Res. 106, 725 (2015)

[5] T. Schmoelzer, K.-D. Liss, G. A. Zickler, I. J. Watson, L. M. Droessler, W. Wallgram, T. Buslaps, A. Studer, and H. Clemens, Intermetallics 18, 1544 (2010).

[6] D. Holec, N. Abdoshahi, S. Mayer, and H. Clemens, Materials 12, 1292 (2019).

[7] S. Semiatin, V. Seetharaman, and I. Weiss, Mater. Sci. Eng. A 243, 1 (1998).

[8] T. Tetsui, K. Shindo, S. Kobayashi, and M. Takeyama, Scr. Mater. 47, 399 (2002).

[9] M. Takeyama and S. Kobayashi, Intermetallics 13, 993 (2005).
[10] H. Clemens, W. Wallgram, S. Kremmer, V. Güther, A. Otto, and A. Bartels, Adv. Eng. Mater. 10, 707 (2008).

[11] S. Mayer, P. Erdely, F. D. Fischer, D. Holec, M. Kastenhuber, T. Klein, and H. Clemens, Adv. Eng. Mater. 19, 1600735 (2017).

[12] T. Schmoelzer, S. Mayer, C. Sailer, F. Haupt, V. Guether, P. Staron, K.-D. Liss, and H. Clemens, Adv. Eng. Mater. 13, 306 (2011).

[13] M. Kimura and K. Hashimoto, J. Phase Equilib. 20, 224 (1999).

[14] R. Kainuma, Y. Fujita, H. Mitsui, I. Ohnuma, and K. Ishida, Intermetallics 8, 855 (2000).

[15] D. Holec, D. Legut, L. Isaeva, P. Souvatzis, H. Clemens, and S. Mayer, Intermetallics 61, 85 (2015).

[16] P. Alippi, P. M. Marcus, and M. Scheffler, Phys. Rev. Lett. 78, 3892 (1997).

[17] V. Paidar, L. G. Wang, M. Šob, and V. Vitek, Modell. Simul. Mater. Sci. Eng. 7, 369 (1999).

[18] M. Šob, L. G. Wang, and V. Vitek, Comput. Mater. Sci. 8, 100 (1997). 
[19] M. Šob, M. Friák, D. Legut, J. Fiala, and V. Vitek, Mater. Sci. Eng. A 387, 148 (2004).

[20] M. Friák, A. Schindlmayr, and M. Scheffler, New J. Phys. 9, 5 (2007).

[21] D. Legut, M. Friák, and M. Šob, Phys. Rev. Lett. 99, 016402 (2007).

[22] D. Legut, M. Friák, and M. Šob, Phys. Rev. B 81, 214118 (2010).

[23] M. Zelený, M. Friák, and M. Šob, Phys. Rev. B 83, 184424 (2011).

[24] M. Friák, T. Hickel, F. Körmann, A. Udyansky, A. Dick, J. von Pezold, D. Ma, O. Kim, W. Counts, M. Šob, T. Gebhardt, D. Music, J. Schneider, D. Raabe, and J. Neugebauer, Steel Res. Int. 82, 86 (2011).

[25] P. J. Craievich, M. Weinert, J. M. Sanchez, and R. E. Watson, Phys. Rev. Lett. 72, 3076 (1994).

[26] M. Šob, L. G. Wang, and V. Vitek, Mater. Sci. Eng. A 234-236, 1075 (1997).

[27] M. Šob, L. G. Wang, and V. Vitek, Philos. Mag. B 78, 653 (1998).

[28] M. Šob, L. G. Wang, and V. Vitek, Kovove Materialy (Metallic Materials) 36, 145 (1998).

[29] M. Friák, M. Šob, and V. Vitek, Phys. Rev. B 63, 052405 (2001).

[30] L. G. Wang, M. Šob, and Z. Zhang, J. Phys. Chem. Solids 64, 863 (2003).

[31] M. Zouhar and M. Šob, Phys. Rev. B 94, 184110 (2016).

[32] G. Kresse and J. Furthmüller, Phys. Rev. B 54, 11169 (1996).

[33] P. E. Blöchl, Phys. Rev. B 50, 17953 (1994).

[34] G. Kresse and D. Joubert, Phys. Rev. B 59, 1758 (1999).

[35] J. P. Perdew, K. Burke, and M. Ernzerhof, Phys. Rev. Lett. 77, 3865 (1996).

[36] A. Zunger, S. H. Wei, L. G. Ferreira, and J. E. Bernard, Phys. Rev. Lett. 65, 353 (1990).

[37] S.-H. Wei, L. G. Ferreira, J. E. Bernard, and A. Zunger, Phys. Rev. B 42, 9622 (1990).

[38] D. Nöger, Optimised structural models of solid solutions for tensorial properties, Master's thesis, Montanuniversität Leoben, Austria, 2017.

[39] T. Chakraborty, J. Rogal, and R. Drautz, Phys. Rev. B 94, 224104 (2016).

[40] M. Friák, M. Všianská, D. Holec, and M. Šob, IOP Conf. Ser.: Mater. Sci. Eng. 219, 012019 (2017).
[41] R. Yu, J. Zhu, and H. Ye, Comput. Phys. Commun. 181, 671 (2010).

[42] M. Moakher and A. N. Norris, J. Elast. 85, 215 (2006).

[43] F. Birch, Phys. Rev. 71, 809 (1947).

[44] C. Jiang, Acta Mater. 56, 6224 (2008).

[45] D. Holec, R. K. Reddy, T. Klein, and H. Clemens, J. Appl. Phys. 119, 205104 (2016).

[46] P. Erdely, P. Staron, A. Stark, T. Klein, H. Clemens, and S. Mayer, Acta Mater. 164, 110 (2019).

[47] M. Musi, P. Erdely, B. Rashkova, H. Clemens, A. Stark, P. Staron, N. Schell, and S. Mayer, Mater. Charact. 147, 398 (2019).

[48] S. Mayer, M. Petersmann, F. D. Fischer, H. Clemens, T. Waitz, and T. Antretter, Acta Mater. 115, 242 (2016).

[49] P. J. Craievich, J. M. Sanchez, R. E. Watson, and M. Weinert, Phys. Rev. B 55, 787 (1997).

[50] J. von Pezold, A. Dick, M. Friák, and J. Neugebauer, Phys. Rev. B 81, 094203 (2010).

[51] Y. Li and M. Loretto, Acta Metall. Mater. 42, 2913 (1994).

[52] A. Singh and D. Banerjee, Metall. Mater. Trans. A 28, 1745 (1997).

[53] S. Azad, R. Mandal, and A. Singh, Mater. Sci. Eng. A 429, 219 (2006).

[54] D. M. Cupid, O. Fabrichnaya, F. Ebrahimi, and H. J. Seifert, Intermetallics 18, 1185 (2010).

[55] S. Mayer, C. Sailer, T. Schmoelzer, H. Clemens, T. Lippmann, P. Staron, V. Güther, and M. Takeyama, BHM Berg-und Hüttenmännische Monatshefte 156, 438 (2011).

[56] V. Witusiewicz, A. Bondar, U. Hecht, O. Stryzhyboroda, N. Tsyganenko, V. Voblikov, V. Petyukh, and T. Y. Velikanova, J. Alloys Compd. 749, 1071 (2018).

[57] S. Kabra, K. Yan, S. Mayer, T. Schmoelzer, M. Reid, R. Dippenaar, H. Clemens, and K.-D. Liss, Int. J. Mater. Res. 102, 697 (2011).

[58] L. Usategui, M. Nó, S. Mayer, H. Clemens, and J. San Juan, Mater. Sci. Eng. A 700, 495 (2017).

[59] K. Momma and F. Izumi, Commission on Crystallogr. Comput. , IUCr Newslett., 7, 106 (2006).

[60] K. Momma and F. Izumi, J. Appl. Crystallogr. 41, 653 (2008).

[61] K. Momma and F. Izumi, J. Appl. Crystallogr. 44, 1272 (2011). 